\title{
Personalized individual semantics in Computing with Words for supporting linguistic Group Decision Making. An Application on Consensus reaching
}

\author{
Cong-Cong $\mathrm{Li}^{a}$, Yucheng Dong ${ }^{a, *}$, Francisco Herrera ${ }^{b, d}$, Enrique \\ Herrera-Viedma ${ }^{b, e}$, and Luis Martínez ${ }^{c, *}$ \\ ${ }^{a}$ Business School, Sichuan University, Chengdu, China \\ ${ }^{b}$ Department of Computer Science and Artificial Intelligence, University of Granada, \\ Granada, Spain \\ ${ }^{c}$ Department of Computer Science, University of Jaén, Jaén, Spain \\ ${ }^{d}$ Faculty of Computing and Information Technology, King Abdulaziz University, North \\ Jeddah, Saudi Arabia \\ ${ }^{e}$ Department of Electrical and Computer Engineering, Faculty of Engineering, King \\ Abdulaziz University, Jeddah, Saudi Arabia
}

\begin{abstract}
In group decision making (GDM) dealing with Computing with Words (CW) has been highlighted the importance of the statement, words mean different things for different people, because of its influence in the final decision. Different proposals that either grouping such different meanings (uncertainty) to provide one representation for all people or use multi-granular linguistic term sets with the semantics of each granularity, have been developed and applied in the specialized literature. Despite these models are quite useful they do not model individually yet the different meanings of each person when he/she elicits linguistic information. Hence, in this paper a personalized individual semantics (PIS) model is proposed to personalize individual semantics by means of an interval numerical scale and the 2-tuple linguistic model. Specifically, a consistency-driven optimization-based model to obtain and represent the PIS is introduced. A new CW framework based on the 2-tuple linguistic model is then defined, such a CW framework allows us to

\footnotetext{
${ }^{*}$ Corresponding authors

E-mail address: congcongli@stu.scu.edu.cn (C. Li), ycdong@scu.edu.cn (Y. Dong),
} herrera@decsai.ugr.es (F. Herrera), viedma@decsai.ugr.es (E. Herrera-Viedma), martin@ujaen.es (L. Martínez)
\end{abstract}


deal with PIS to facilitate CW keeping the idea that words mean different things to different people. In order to justify the feasibility and validity of the PIS model, it is applied to solve linguistic GDM problems with a consensus reaching process.

Keywords: computing with words, 2-tuple linguistic model, semantics, group decision making, preference relations

\section{Introduction}

Human beings usually employ words in most of their computing and reasoning processes without the necessity of any precise number. Computing with words $(\mathrm{CW})$ is a methodology in which the objects of computation are words and propositions drawn from a natural language $[49,50]$ that arises to emulate such human behaviors. Hence a crucial feature of $\mathrm{CW}$ is that its processes deal with linguistic inputs to obtain linguistic outputs easy to understand by human beings. Different computing schemes have been proposed for CW that could be summarized in Fig. 1. Yager [48] points out the importance of the translation and retranslation processes to achieve the aims of the CW.

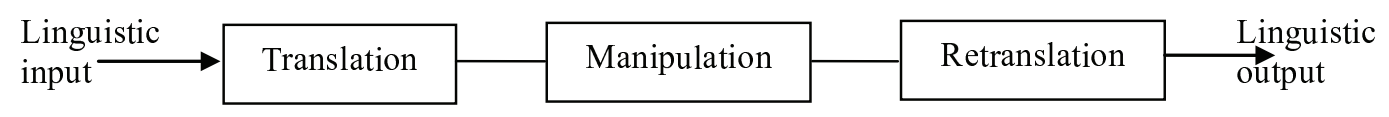

Fig. 1. Yager's CW scheme

It is important to remark that $\mathrm{CW}$ involves a wide-ranging ramifications and applications from learning to decision making passing by many others $[23,13,15,39,40]$. Our interest in this paper is focused on the use of $\mathrm{CW}$ in decision making [28]. Specifically on group decision making (GDM) because its use implies another key and controversial point about CW, that it is the fact that words mean different things for different people [1, 16, 29, 30]. In order to deal with previous fact that increases the difficulty of managing the uncertainty of linguistic information, two mainstreams have been developed in the literature: 
1. The use of type-2 fuzzy sets based on low and upper possibility distributions with a third dimension in between [29], that group all meanings from people in just one representation function and,

2. The use of multi-granular linguistic models $[14,19,33]$ in which multiple linguistic term sets can be used by experts according to either their degree of knowledge or their comfort or their similarity with the semantics of each granularity.

In spite of both previous methods are quite useful to deal with the multiple meanings of words and have been also widely used for $\mathrm{CW}$ in multiple different problems, they do not represent yet the specific semantics of each individual. For example, when reviewing an article, two referees both think this article is "Good", but the term "Good" often has different numerical meaning for these two referees. Hence, in this paper a personalized individual semantics (PIS) model is proposed to customize individual semantics by means of an interval numerical scale $[6,12]$ and the 2-tuple linguistic model [18]. In order to do so, this paper develops two main proposals:

a) A new model to represent PIS, such that it will be based on the interval numerical scale because of its features to deal with different linguistic representations in a precise way $[6,12]$.

b) A framework for CW dealing with PIS, based on the 2-tuple linguistic model [27], including personalized 2-tuple linguistic operators are proposed, because of its good features for managing linguistic information in CW processes [38]. This framework will cope with PIS and redesign the CW phases pointed out in Fig. 1 to obtain customized accurate linguistic results easy to interpret and understand by individuals.

There are a lot of researches regarding GDM problems using linguistic preference relations, such as aggregation operators [3], consistency measures $[8,10]$, consensus models $[9,20,34]$ and so on. In order to justify the feasibility and validity of the PIS model, it will be applied to a linguistic GDM problem with a consensus reaching process, by defining the concept of the individual linguistic understanding.

The remainder of this paper is arranged as follows. Section 2 introduces a basic description of the 2-tuple linguistic model, the numerical scale and 
preference relations. Section 3 introduces a consistency-driven optimizationbased model to obtain the interval numerical scale of PIS for decision makers in linguistic GDM problems. Section 4 proposes a new CW framework based on the 2-tuple linguistic model for dealing with PIS. Section 5 presents a consensus reaching process for linguistic GDM problems with PIS. Section 6 then concludes this paper.

\section{Preliminaries}

This section introduces the basic necessary knowledge to understand our proposals, regarding the 2-tuple linguistic model, the numerical scale and preference relations.

\subsection{The 2-tuple linguistic model}

The 2-tuple linguistic representation model, presented in Herrera and Martínez [18] represents the linguistic information by a 2-tuple $\left(s_{i}, \alpha\right) \in \bar{S}=$ $S \times[-0.5,0.5)$, where $s_{i} \in S$ and $\alpha \in[-0.5,0.5)$. Formally, let $S=\left\{s_{i} \mid i=\right.$ $0,1,2, \ldots, g\}$ be a linguistic term set and $\beta \in[0, g]$ be a value representing the result of a symbolic aggregation operation. The 2-tuple that expresses the equivalent information to $\beta$ is then obtained as:

$$
\Delta:[0, g] \rightarrow S \times[-0.5,0.5)
$$

where

$$
\Delta(\beta)=\left(s_{i}, \alpha\right) \text {, with }\left\{\begin{array}{c}
s_{i}, i=\operatorname{round}(\beta) \\
\alpha=\beta-i, \alpha \in[-0.5,0.5)
\end{array} .\right.
$$

Function $\Delta$, it is a one to one mapping whose inverse function $\Delta^{-1}: \bar{S} \rightarrow$ $[0, g]$ is defined as $\Delta^{-1}\left(s_{i}, \alpha\right)=i+\alpha$. When $\alpha=0$ in $\left(s_{i}, \alpha\right)$ is then called simple term.

In [18] it was also defined a computational model for linguistic 2-tuples in which different operations were introduced:

(1) A 2-tuple comparison operator: Let $\left(s_{k}, \alpha\right)$ and $\left(s_{l}, \gamma\right)$ be two 2-tuples. Then:

(i) if $k<l$, then $\left(s_{k}, \alpha\right)$ is smaller than $\left(s_{l}, \gamma\right)$.

(ii) if $k=l$, then

(a) if $\alpha=\gamma$, then $\left(s_{k}, \alpha\right),\left(s_{l}, \gamma\right)$ represents the same information.

(b) if $\alpha<\gamma$, then $\left(s_{k}, \alpha\right)$ is smaller than $\left(s_{l}, \gamma\right)$.

(2) A 2-tuple negation operator: 
$\operatorname{Neg}\left(\left(s_{i}, \alpha\right)\right)=\Delta\left(g-\left(\Delta^{-1}\left(s_{i}, \alpha\right)\right)\right)$.

(3) Several 2-tuple aggregation operators have been developed (see [18, $31])$.

\subsection{Numerical scale to extend the 2-tuple linguistic model}

Dong et al. [11, 12] extended the 2-tuple linguistic model by the numerical scale and the interval numerical scale for integrating different linguistic models and increasing the accuracy of the 2-tuple linguistic computational model.

(1) Numerical scale

The concept of the numerical scale was introduced in [11] for transforming linguistic terms into real numbers:

Definition 1. [11] Let $S=\left\{s_{i} \mid i=0,1,2, \ldots, g\right\}$ be a linguistic term set, and $R$ be the set of real numbers. The function: $N S: S \rightarrow R$ is defined as a numerical scale of $S$, and $N S\left(s_{i}\right)$ is called the numerical index of $s_{i}$. If the function NS is strictly monotone increasing, then NS is called an ordered numerical scale.

Definition 2. [11] Let $S, \bar{S}$ and $N S$ be as before. The numerical scale $\overline{N S}$ on $\bar{S}$ for $\left(s_{i}, \alpha\right) \in \bar{S}$, is defined by

$$
\overline{N S}\left(\left(s_{i}, \alpha\right)\right)=\left\{\begin{array}{ll}
N S\left(s_{i}\right)+\alpha \times\left(N S\left(s_{i+1}\right)-N S\left(s_{i}\right)\right), & \alpha \geq 0 \\
N S\left(s_{i}\right)+\alpha \times\left(N S\left(s_{i}\right)-N S\left(s_{i-1}\right)\right), & \alpha<0
\end{array} .\right.
$$

To simplify the notation, $\overline{N S}$ will also be denoted as $N S$ in this paper.

In [11] $N S$ was introduced as a a family of functions, that usually are ordered functions, if so it was proved that its inverse $N S^{-1}$ exists. For example, setting $N S\left(s_{i}\right)=\Delta^{-1}\left(s_{i}\right)$ (i.e., $N S\left(s_{0}\right)=0, N S\left(s_{1}\right)=1, \ldots, N S\left(s_{g}\right)=g$ ) yields the 2-tuple linguistic model [18].

(2) Interval numerical scale

The concept of the interval numerical scale [12] extends the numerical scale model to transform linguistic terms into numerical interval values:

Definition 3. [12] Let $S=\left\{s_{i} \mid i=0,1,2, \ldots, g\right\}$ be a linguistic term set, and let $M=\left\{\left[A_{L}, A_{R}\right] \mid A_{L}, A_{R} \in[0,1], A_{L} \leq A_{R}\right\}$ be a set of interval values in $[0,1]$. The function INS:S $\rightarrow M$ is defined as an interval numerical scale of $S$, and $I N S\left(s_{i}\right)$ is called the interval numerical index of $s_{i}$. 
If $I N S\left(s_{i}\right)=\left[A_{L}^{i}, A_{R}^{i}\right]$, then the functions $I N S_{L}$ and $I N S_{R}$ are defined as follows: $I N S_{L}\left(s_{i}\right)=A_{L}^{i}$ and $I N S_{R}\left(s_{i}\right)=A_{R}^{i}$. The interval numerical scale $I N S$ is ordered if $I N S_{L}\left(s_{i}\right)<I N S_{L}\left(s_{i+1}\right)$ and $I N S_{R}\left(s_{i}\right)<I N S_{R}\left(s_{i+1}\right)$ for $i=0,1, \ldots, g-1$.

Definition 4. [12] Let $S, \bar{S}$ and $N S$ be as before. For $\left(s_{i}, \alpha\right) \in \bar{S}$, the interval numerical scale INS on $\bar{S}$ is defined by

$$
\operatorname{INS}\left(\left(s_{i}, \alpha\right)\right)=\left[A_{L}, A_{R}\right],
$$

where

$$
\begin{gathered}
A_{L}=\left\{\begin{array}{cc}
I N S_{L}\left(s_{i}\right)+\alpha \times\left(I N S_{L}\left(s_{i+1}\right)-I N S_{L}\left(s_{i}\right)\right), & \alpha \geq 0 \\
I N S_{L}\left(s_{i}\right)+\alpha \times\left(I N S_{L}\left(s_{i}\right)-I N S_{L}\left(s_{i-1}\right)\right), & \alpha<0
\end{array}\right. \\
A_{R}=\left\{\begin{array}{cc}
I N S_{R}\left(s_{i}\right)+\alpha \times\left(I N S_{R}\left(s_{i+1}\right)-I N S_{R}\left(s_{i}\right)\right), & \alpha \geq 0 \\
I N S_{R}\left(s_{i}\right)+\alpha \times\left(I N S_{R}\left(s_{i}\right)-I N S_{R}\left(s_{i-1}\right)\right), & \alpha<0
\end{array} .\right.
\end{gathered}
$$

Dong et al. [12] introduced the inverse operation of $I N S$ noted as $I N S^{-1}$ and its generalization, a simplified inverse operation $I N S^{-1}$ is defined as:

Definition 5. Let $S=\left\{s_{i} \mid i=0,1,2, \ldots, g\right\}$ be a linguistic term set, INS be an ordered interval numerical scale on $\bar{S}$, and $M=\left\{\left[A_{L}, A_{R}\right] \mid A_{L}, A_{R} \in\right.$ $\left.[0,1], A_{L} \leq A_{R}\right\}$ a set of interval values in $[0,1]$. The inverse operation $I N S^{-1}$ is defined as:

$$
I N S^{-1}: M \longrightarrow \bar{S}
$$

where for any $A \in M, I N S^{-1}(A)=s$ and

$$
d(A, I N S(s))=\min _{x \in \bar{S}} d(A, I N S(x)) .
$$

In Eq.(8), $d$ is a distance function for interval values. Different distance functions might be applied to computing $I N S^{-1}$, and in this paper it is used the Euclidean distance, i.e., $d([a, b],[c, d])=\sqrt{(a-c)^{2}+(b-d)^{2}}$, because it provides correct results and is generally utilized in the retranslation process in $\mathrm{CW}$ [48].

In [12] was also introduced a way to compute $I N S$ and $I N S^{-1}$.

Example 1. Let $S=\left\{s_{0}, s_{1}, \ldots, s_{4}\right\}$. Let $I N S\left(s_{0}\right)=[0,0.1], \operatorname{IN} S\left(s_{1}\right)=$ $[0.2,0.25], \operatorname{INS}\left(s_{2}\right)=0.5, \operatorname{INS}\left(s_{3}\right)=[0.75,0.8]$, and $\operatorname{IN} S\left(s_{4}\right)=[0.751,1]$. 
(1) Hence the interval numerical index of a linguistic 2-tuple $\left(s_{1}, 0.3\right)$ is $I N S\left(\left(s_{1}, 0.3\right)\right)=\left[A_{L}, A_{R}\right]$ and according to Eq.(5):

$A_{L}=I N S_{L}\left(s_{1}\right)+0.3 \times\left(I N S_{L}\left(s_{2}\right)-I N S_{L}\left(s_{1}\right)\right)=0.2+0.3 \times(0.5-0.2)=0.29$.

Moreover, according to Eq.(6):

$A_{R}=I N S_{R}\left(s_{1}\right)+0.3 \times\left(I N S_{R}\left(s_{2}\right)-I N S_{R}\left(s_{1}\right)\right)=0.25+0.3 \times(0.5-0.25)=$ 0.325 .

Consequently, INS $\left(\left(s_{1}, 0.3\right)\right)=\left[A_{L}, A_{R}\right]=[0.29,0.325]$.

(2) To illustrate how to obtain the value of $I N S^{-1}([0.6,0.8])$ it must be used Eqs. (7) and (8):

$\min _{x \in \bar{S}} d([0.6,0.8], I N S(s))=d\left([0.6,0.8], I N S\left(s_{3},-0.246\right)\right)=0.013$.

Therefore, $I N S^{-1}([0.6,0.8])=\left(s_{3},-0.246\right)$.

In the linguistic computational model with the interval numerical scale, the input are linguistic terms, and the output are 2-tuple linguistic intervals to avoid the loss of information. Further detail regarding the operations with the interval numerical scale can be found in [12].

Remark 1[7, 11]. The numerical scale can provide a connection among the 2-tuple linguistic model and its variants, additionally can set different numerical scales for the 2-tuple linguistic model [18], the Wang and Hao model [41] and the unbalanced linguistic model based on a linguistic hierarchy [17].

Remark 2[11]. The interval numerical scale can be reduced to the numerical scale. So the interval numerical scale will be used as the basis to develop the 2-tuple linguistic model with PIS in this paper.

\subsection{Linguistic and numerical preference relations. Consistency}

Let $X=\left\{X_{1}, X_{2}, \ldots, X_{m}\right\}(n \geq 2)$ be a finite set of alternatives. When a decision maker provides pairwise comparisons using the linguistic term set $S$, he/she can construct a linguistic preference relation $L=\left(l_{i j}\right)_{n \times n}$, whose element $l_{i j}$ estimates the preference degree of alternative $X_{i}$ over $X_{j}$. Linguistic preference relations based on linguistic 2-tuples can be formally defined as:

Definition 6. [2] The matrix $L=\left(l_{i j}\right)_{n \times n}$, where $l_{i j} \in S$, is called a simple linguistic preference relation. The matrix $L=\left(l_{i j}\right)_{n \times n}$, where $l_{i j} \in \bar{S}$, is called a 2-tuple linguistic preference relation. If $l_{i j}=N e g\left(l_{j i}\right)$ for $i, j=1,2, \ldots, n$, then $L$ is considered reciprocal. 
In addition, the numerical preference relations are often used in decision making. A kind of numerical preference relations, i.e., fuzzy preference relations were also introduced.

Definition 7. [22, 36] The matrix $F=\left(f_{i j}\right)_{n \times n}$, where $f_{i j} \in[0,1]$ and $f_{i j}+f_{j i}=1$ for $i, j=1,2, \ldots, n$, is called a fuzzy preference relation.

The study of consistency in a preference relation is very important, because it ensures that preferences are neither random nor illogical. Generally, ordinal [45] and cardinal [4] consistency are two common types of consistency for a preference relation. The former is closely related to the transitivity of the corresponding preference relation meanwhile the latter is a stronger concept because it not only implies the transitivity of preferences, but also the intensity of preference expressed by comparisons. Here, it is revised the cardinal consistency index (CI) based on additive transitivity [21] for fuzzy preference relations, $F$, because it will be extended in our proposal:

$$
C I(F)=1-\frac{2}{3 n(n-1)(n-2)} \sum_{i, j, k=1 ; i \neq j \neq k}^{n}\left|f_{i j}+f_{j k}-f_{i k}-0.5\right| .
$$

Due to the complexity and uncertainty involved in real-world decision problems, sometimes it is unrealistic to acquire exact judgments. Thus, fuzzy preference relations are extended to interval fuzzy preference relations.

Definition 8. [46] The matrix $\widetilde{V}=\left(\widetilde{v_{i j}}\right)_{n \times n}$, where $\widetilde{v_{i j}}=\left[v_{i j}^{-}, v_{i j}^{+}\right] \subseteq[0,1]$ and $v_{i j}^{-}+v_{j i}^{+}=1$ for $i, j=1,2, \ldots, n$, is called an interval fuzzy preference relation.

Definition 9. [5] Let $\widetilde{V}=\left(\widetilde{v_{i j}}\right)_{n \times n}$, where $\widetilde{v_{i j}}=\left[v_{i j}^{-}, v_{i j}^{+}\right]$, be an interval fuzzy preference relation. $F=\left(f_{i j}\right)_{n \times n}$ is a fuzzy preference relation associated to $\widetilde{V}$ if $v_{i j}^{-} \leq f_{i j} \leq v_{i j}^{+}$and $f_{i j}+f_{j i}=1$.

Being $N_{\widetilde{V}}$ the set of the fuzzy preference relations associated to $\widetilde{V}$.

Remark 3. Reciprocity is an important property of preference relations. However, when $\mathrm{S}$ is not uniformly and symmetrically distributed, the reciprocity of linguistic preference relations cannot be guaranteed. In this situation, it is assumed that the decision maker only provides his/her preferences for the upper/lower triangular entries of $L$. 


\section{Personalized individual semantics based on interval numerical scales}

As aforementioned, the difficulty of carrying out CW processes with the issue words mean different things for different people that naturally arises in problems with multiple experts like GDM problems still remains open. Even though, different proposals have been introduced in the literature based on type-1 [14] and type-2 [29] fuzzy sets, dealing with multiple linguistic term sets and grouping individual representations respectively. In fact, neither of them represents specifically the PIS of each expert involved in the GDM problem.

Therefore, the first objective of this paper is to introduce an interval numerical scale based method to personalize individual semantics represented by interval values from the linguistic preference relations elicited by the experts taking part in the GDM problem. This representation will be managed in the CW framework presented later in Section 4.

The method to obtain the PIS consists of a consistency-driven optimization model. Before introducing this model it is necessary to fix some notations, premises and a consistency measure for interval fuzzy preference relations introduced in the coming subsections.

\subsection{Basics}

Let $S=\left\{s_{i} \mid i=0,1, \ldots, g\right\}$ be a linguistic term set, $I N S^{k}$ be an ordered interval numerical scale on $S$ associated with the individual $e_{k}(k=1,2, \ldots, m)$, and $L^{k}=\left(l_{i j}^{k}\right)_{n \times n}$ be the linguistic preference relation based on $S$ associated with $e_{k}$. The matrix $\widetilde{V^{k}}=\left(\widetilde{v_{i j}^{k}}\right)_{n \times n}$, in which $v_{i j}^{k}=\left[v_{i j}^{k-}, v_{i j}^{k+}\right]=I N S^{k}\left(l_{i j}^{k}\right)$, is called the numerical preference relation transformed by $I N S^{k}$, associated with $L^{k}$.

Remark 3 pointed out that when an individual only provides his/her preference information for the upper/lower triangular entries of the linguistic preference relations based on $\mathrm{S}$, the reciprocity of the numerical preference relation $\widetilde{V^{k}}$ will not be violated. Besides, in the 2-tuple linguistic model with the interval numerical scale, the support of the $I N S^{k}$ of $S$ is the interval $[0,1]$. As a result, $\widetilde{V^{k}}$ is an interval fuzzy preference relation. Hence, $L^{k}$ and $\widetilde{V^{k}}$ represent the same preference, associated with $e_{k}$. So, $\widetilde{V^{k}}$ should be consistent if $L^{k}$ is consistent. From this reasoning in [6] was provided the following premise: 
Premise 1 [6]. If linguistic preference relations provided by individuals are consistent, then the interval fuzzy preference relations, transformed by the established interval numerical scales, should be as much consistent as possible.

The Premise 1 implies the need of consistency in interval fuzzy preference relations, and the ordinal consistency can be guaranteed by the transformation from linguistic to interval fuzzy preference relations. However, the cardinal consistency should be still studied with a specific measure, and here we propose the cardinal interval consistency index based on Eq. (9):

Definition 10. Let $\widetilde{V}=\left(\widetilde{v_{i j}}\right)_{n \times n}$ be an interval fuzzy preference relation, let $F=\left(f_{i j}\right)_{n \times n}$ be a fuzzy preference relation associated to $\widetilde{V}$, and let $N_{\widetilde{V}}$ be the set of the fuzzy preference relation associated to $\widetilde{V}$. The optimistic consistency index (OCI) of $\widetilde{V}$ is then defined as follows,

$$
O C I(\widetilde{V})=\max _{F \in N_{\widetilde{V}}} C I(F)
$$

i.e.,

$$
O C I(\widetilde{V})=\max _{F \in N_{\tilde{V}}}\left(1-\frac{2}{3 n(n-1)(n-2)} \sum_{i, j, z=1 ; i \neq j \neq z}^{n}\left|f_{i j}+f_{j z}-f_{i z}-0.5\right|\right),
$$

and the pessimistic consistency index (PCI) of $\widetilde{V}$ is,

$$
\begin{aligned}
& \operatorname{PCI}(\widetilde{V})=\min _{F \in N_{\tilde{V}}} C I(F), \\
& \text { i.e., } \\
& P C I(\widetilde{V})=\min _{F \in N_{\tilde{V}}}\left(1-\frac{2}{3 n(n-1)(n-2)} \sum_{i, j, z=1 ; i \neq j \neq z}^{n}\left|f_{i j}+f_{j z}-f_{i z}-0.5\right|\right) .
\end{aligned}
$$

In the proposed interval consistency index, $O C I(\widetilde{V})$ and $\operatorname{PCI}(\widetilde{V})$ reflect the best and worst consistency indexes of all fuzzy preference relations associated to $\widetilde{V}$, respectively.

In previous studies regarding the consistency measure of $\widetilde{V}$ (e.g., [6, 42]), $\operatorname{OCI}(\widetilde{V})$ was considered as the consistency degree of $\widetilde{V}$. However, $\operatorname{OCI}(\widetilde{V})$ 
cannot accurately measure the consistency degree of $\widetilde{V}$ such as it is illustrated in Example 2.

Example 2. Consider the following interval fuzzy preference relation:

$$
\widetilde{V}=\left(\begin{array}{ccc}
{[0.5,0.5]} & {[0.2,1]} & {[0.1,0.3]} \\
{[0,0.8]} & {[0.5,0.5]} & {[0.3,0.9]} \\
{[0.7,0.9]} & {[0.1,0.7]} & {[0.5,0.5]}
\end{array}\right)
$$

Solving Eq. (10) obtains $\max _{F \in N_{\tilde{V}}} C I(F)=C I\left(F^{1}\right)=1$, where

$$
F^{1}=\left(\begin{array}{ccc}
0.5 & 0.5 & 0.3 \\
0.5 & 0.5 & 0.3 \\
0.7 & 0.7 & 0.5
\end{array}\right)
$$

so, $O C I(\widetilde{V})=C I\left(F^{1}\right)=1$. Solving Eq. (12) obtains $\min _{F \in N_{\tilde{V}}} C I(F)=$ $C I\left(F^{2}\right)=0.133$, in which

$$
F^{2}=\left(\begin{array}{ccc}
0.5 & 1 & 0.1 \\
0 & 0.5 & 0.9 \\
0.9 & 0.1 & 0.5
\end{array}\right)
$$

so, $P C I(\widetilde{V})=C I\left(F^{2}\right)=0.133$.

In Example 2, $F^{1}$ reflects the best consistency degree of $\widetilde{V}$, and $F^{2}$ reflects the worst consistency degree of $\widetilde{V}$. Besides, $O C I(\widetilde{V})=1$, i.e., $\widetilde{V}$ is fully consistent based on $O C I(\widetilde{V})$. But, $P C I(\widetilde{V})=0.133$ is very low. Hence, $O C I(\widetilde{V})$ cannot accurately measure the consistency degree of $\widetilde{V}$.

Remark 4. Clearly, the consistency index $C I$ of any fuzzy preference relation $F$ associated to $\widetilde{V}$ is in the interval $[P C I(\widetilde{V}), O C I(\widetilde{V})]$, i.e., $C I(F) \in$ $[P C I(\widetilde{V}), O C I(\widetilde{V})]$ for any $F \in N_{\widetilde{V}}$.

Therefore our proposal will use $O C I(\widetilde{V})$ and $P C I(\widetilde{V})$ because they reflect better the consistency degree of $\widetilde{V}$ than just the use of $O C I(\widetilde{V})$.

\subsection{A consistency-driven optimization-based model to obtain personalized individual semantics}

From our view the personal own meaning (semantics) that each individual provides to words when eliciting linguistic preferences are closely related 
to her/his consistency. Therefore this section introduces a consistency-driven optimization-based model to obtain the personalized individual interval numerical scales of the 2-tuple linguistic terms.

Let $I N S^{k}\left(s_{i}\right)=\left[A_{L}^{i, k}, A_{R}^{i, k}\right]$ be the interval numerical index of $s_{i}$, associated with the decision maker $e_{k}$. According to Premise 1 , if $L^{k}$ is consistent, then $\widetilde{V^{k}}$ should be as much consistent as possible. It is then necessary to maximize $\operatorname{PCI}\left(\widetilde{V^{k}}\right)$ by,

$$
\max \min C I\left(F^{2 k}\right)
$$

where

$$
F^{2 k} \in N_{\widetilde{V^{k}}}, k=1,2, \ldots, m .
$$

In the previous studies $[6,42]$ it was required that $O C I\left(\widetilde{V^{k}}\right)=1$, so

$$
C I\left(F^{1 k}\right)=1, k=1,2, \ldots, m
$$

where

$$
F^{1 k} \in N_{\widetilde{V^{k}}}, k=1,2, \ldots, m .
$$

Based on the existing several 2-tuple linguistic models (e.g., the Herrera and Martínez model [18], the Wang and Hao model [41], and the unbalanced linguistic model [17]), the ordered initial numerical index $a_{i}$ of $s_{i}$ can be provided by different functions that computes $N S$. For example, in the Herrera and Martínez model, $a_{i}=N S\left(s_{i}\right)=\Delta^{-1}\left(s_{i}\right) / g$; in the Wang and Hao model, $N S\left(s_{i}\right)$ is determined by canonical characteristic values; in the unbalanced linguistic model, $N S\left(s_{i}\right)$ is determined by a linguistic hierarchy. This paper assumes that $a_{i} \in I N S^{k}\left(s_{i}\right)$, i.e.,

$$
0 \leq A_{L}^{i, k} \leq a_{i} \leq A_{R}^{i, k} \leq 1, i=0,1, \ldots, g ; k=1,2, \ldots, m
$$

Moreover, $I N S^{k}$ is ordered, then:

$$
I N S_{L}^{k}\left(s_{i}\right)<I N S_{L}^{k}\left(s_{i+1}\right), i=0,1, \ldots, g-1 ; k=1,2, \ldots, m,
$$

and

$$
I N S_{R}^{k}\left(s_{i}\right)<I N S_{R}^{k}\left(s_{i+1}\right), i=0,1, \ldots, g-1 ; k=1,2, \ldots, m .
$$

Based on Eqs. (14)-(20), an optimization model to set individual interval numerical scales of linguistic terms $I N S^{k}\left(s_{i}\right)=\left[A_{L}^{i, k}, A_{R}^{i, k}\right]$ can be constructed as follows, 


$$
\left\{\begin{array}{l}
\max \min C I\left(F^{2 k}\right) \\
\text { s.t. } F^{2 k} \in N_{\widetilde{V^{k}}}, \quad k=1,2, \ldots, m \\
C I\left(F^{1 k}\right)=1, \quad k=1,2, \ldots, m \\
F^{1 k} \in N_{\widetilde{V^{k}}}, \quad k=1,2, \ldots, m \\
0 \leq A_{L}^{i, k} \leq a_{i} \leq A_{R}^{i, k} \leq 1 \quad i=0,1, \ldots, g ; k=1,2, \ldots, m \\
I N S_{L}^{k}\left(s_{i}\right)<I N S_{L}^{k}\left(s_{i+1}\right) \quad i=0,1, \ldots, g-1 ; k=1,2, \ldots, m \\
I N S_{R}^{k}\left(s_{i}\right)<I N S_{R}^{k}\left(s_{i+1}\right) \quad i=0,1, \ldots, g-1 ; k=1,2, \ldots, m
\end{array}\right.
$$

Remark 5. Using $[P C I(\widetilde{V}), O C I(\widetilde{V})]$ for measuring the consistency degree of $\widetilde{V}$, model $(21)$ sets $O C I\left(\widetilde{V^{k}}\right)=1$ based on the previous studies[6, 42], and the objective function is set to maximize $\operatorname{PCI}\left(\widetilde{V^{k}}\right)$. In this way, $\widetilde{V^{k}}$ can be as much consistent as possible.

Let $I N S^{k}\left(s_{i}\right)=\left[A_{L}^{i, k}, A_{R}^{i, k}\right]$, and let $p(s)$, where $s \in S$, be the position index of $s$. For example, if $s=s_{i}$, then $p(s)=i$. Thus $I N S^{k}\left(l_{i j}^{k}\right)=$ $\left[A_{L}^{p\left(l_{i j}^{k}\right), k}, A_{R}^{p\left(l_{i j}^{k}\right), k}\right]\left(l_{i j}^{k} \neq n u l l\right)$.

Proposition 1. Model (21) can be equivalently transformed into model (22)(30), denoted as $P$.

$$
\max \min 1-\frac{2}{3 n(n-1)(n-2)} \sum_{i, j, z=1 ; i \neq j \neq z}^{n}\left|f_{i j}^{2 k}+f_{j z}^{2 k}-f_{i z}^{2 k}-0.5\right|
$$

s.t.

$$
\begin{aligned}
& A_{L}^{p\left(l_{i j}^{k}\right), k} \leq f_{i j}^{2 k} \leq A_{R}^{p\left(l_{i j}^{k}\right), k}, i, j=1,2, \ldots, n, i \neq j ; k=1,2, \ldots, m ; l_{i j}^{k} \neq \text { null } \\
& f_{i j}^{2 k}+f_{j i}^{2 k}=1, i, j=1,2, \ldots, n, i \neq j ; k=1,2, \ldots, m \\
& f_{i j}^{1 k}+f_{j z}^{1 k}-f_{i z}^{1 k}=0.5, i, j=1,2, \ldots, n, i \neq j ; k=1,2, \ldots, m \\
& A_{L}^{p\left(l_{i j}^{k}\right), k} \leq f_{i j}^{1 k} \leq A_{R}^{p\left(l_{i j}^{k}\right), k}, i, j=1,2, \ldots, n, i \neq j ; k=1,2, \ldots, m ; l_{i j}^{k} \neq n u l l \\
& f_{i j}^{1 k}+f_{j i}^{1 k}=1, i, j=1,2, \ldots, n, i \neq j ; k=1,2, \ldots, m \\
& 0 \leq A_{L}^{i, k} \leq a_{i} \leq A_{R}^{i, k} \leq 1, i=0,1, \ldots, g \\
& A_{L}^{i, k}<A_{L}^{i+1, k}, i=0,1, \ldots, g-1 ; k=1,2, \ldots, m \\
& A_{R}^{i, k}<A_{R}^{i+1, k}, i=0,1, \ldots, g-1 ; k=1,2, \ldots, m
\end{aligned}
$$


The proof of Proposition 1 is provided in Appendix.

Model $P$ can be easily transformed into a max-min linear programming model. By solving $P$, it is obtained the individual interval numerical indexes $I N S^{k}\left(s_{i}\right)=\left[A_{L}^{i, k}, A_{R}^{i, k}\right]$ that reflect in the best possible way the individual meaning of words because it reflects the best consistency in their preferences. According to Miller [32], an individual cannot simultaneously compare more than $7 \pm 2$ objects without producing confusion. So, the size of matrices, i.e., $n$, should be smaller than 9 . As a result, the proposed model $P$ is a small-scale optimization problem, and can be effectively and rapidly solved by several software packages (e.g., Matlab and Lingo).

\subsection{Illustration of the consistency-driven optimization-based model}

The following example illustrates the consistency-driven optimizationbased model.

Example 3. Let's suppose a set of five decision makers, $E=\left\{e_{1}, e_{2}, \ldots, e_{5}\right\}$ and a set of five alternatives, $X=\left\{X_{1}, X_{2}, \ldots, X_{5}\right\}$. Let $S=\left\{s_{0}=\right.$ extremely poorer, $s_{1}=$ much poorer, $s_{2}=$ fair, $s_{3}=$ better, $s_{4}=$ extremely better $\}$ be an established linguistic term set. The decision maker $e_{k}$ supplies the linguistic preference relation based on $S, L^{k}$, to express his/her opinions over $X$. These preference relations $L^{k}(k=1,2, \ldots, 5)$ are listed as follows.

$L^{1}=\left(\begin{array}{ccccc}\text { null } & s_{3} & s_{4} & s_{1} & s_{1} \\ \text { null } & \text { null } & s_{3} & s_{0} & s_{1} \\ \text { null } & \text { null } & \text { null } & s_{0} & s_{0} \\ \text { null } & \text { null } & \text { null } & \text { null } & s_{3} \\ \text { null } & \text { null } & \text { null } & \text { null } & \text { null }\end{array}\right), \quad L^{2}=\left(\begin{array}{ccccc}\text { null } & s_{2} & s_{0} & s_{0} & s_{0} \\ \text { null } & \text { null } & s_{1} & s_{1} & s_{1} \\ \text { null } & \text { null } & \text { null } & s_{2} & s_{1} \\ \text { null } & \text { null } & \text { null } & \text { null } & s_{1} \\ \text { null } & \text { null } & \text { null } & \text { null } & \text { null }\end{array}\right)$,

$L^{3}=\left(\begin{array}{ccccc}\text { null } & s_{3} & s_{0} & s_{1} & s_{1} \\ \text { null } & \text { null } & s_{0} & s_{1} & s_{1} \\ \text { null } & \text { null } & \text { null } & s_{3} & s_{3} \\ \text { null } & \text { null } & \text { null } & \text { null } & s_{1} \\ \text { null } & \text { null } & \text { null } & \text { null } & \text { null }\end{array}\right), \quad L^{4}=\left(\begin{array}{ccccc}\text { null } & s_{2} & s_{1} & s_{0} & s_{0} \\ \text { null } & \text { null } & s_{1} & s_{1} & s_{1} \\ \text { null } & \text { null } & \text { null } & s_{2} & s_{2} \\ \text { null } & \text { null } & \text { null } & \text { null } & s_{2} \\ \text { null } & \text { null } & \text { null } & \text { null } & \text { null }\end{array}\right)$,

$L^{5}=\left(\begin{array}{ccccc}\text { null } & \text { null } & \text { null } & \text { null } & \text { null } \\ s_{2} & \text { null } & \text { null } & \text { null } & \text { null } \\ s_{3} & s_{3} & \text { null } & \text { null } & \text { null } \\ s_{4} & s_{3} & s_{2} & \text { null } & \text { null } \\ s_{4} & s_{4} & s_{3} & s_{3} & \text { null }\end{array}\right)$. 
Without loss of generality, let the initial numerical index $a_{i}$ of $s_{i}$ be $\{0,0.25,0.5,0.75,1\}$. Solving model $P$ obtains the interval numerical indexes that represent the PIS of each decision maker $I N S^{k}\left(s_{i}\right)=\left[A_{L}^{i}, A_{R}^{i}\right]$, which are listed in Table 1.

Table 1. Values of $I N S^{k}\left(s_{i}\right)(k=1,2, \ldots, 5 ; i=0,1, \ldots, 4)$

\begin{tabular}{llllll}
\hline & $I N S^{1}\left(s_{i}\right)$ & $I N S^{2}\left(s_{i}\right)$ & $I N S^{3}\left(s_{i}\right)$ & $I N S^{4}\left(s_{i}\right)$ & $I N S^{5}\left(s_{i}\right)$ \\
\hline$i=0$ & {$[0,0.125]$} & {$[0,0.375]$} & {$[0,0.249]$} & {$[0,0.249]$} & 0 \\
$i=1$ & {$[0.25,0.375]$} & {$[0.25,0.376]$} & {$[0.25,0.499]$} & {$[0.249,0.25]$} & 0.25 \\
$i=2$ & 0.5 & 0.5 & 0.5 & 0.5 & 0.5 \\
$i=3$ & {$[0.625,0.75]$} & {$[0.625,0.75]$} & {$[0.748,0.75]$} & 0.75 & {$[0.75,0.751]$} \\
$i=4$ & {$[0.75,1]$} & {$[0.626,1]$} & 1 & 1 & {$[0.751,1]$} \\
\hline
\end{tabular}

Using the obtained interval numerical scale $I N S^{k}$ transforms $L^{k}$ into the interval fuzzy preference relation $\left.\widetilde{V^{k}}=\widetilde{\left(v_{i j}^{k}\right.}\right)_{5 \times 5}$, where $\widetilde{v_{i j}^{k}}=\left[v_{i j}^{k-}, v_{i j}^{k+}\right](k=$ $1,2, \ldots, 5)$.

$$
\begin{aligned}
& \widetilde{V^{1}}=\left(\begin{array}{ccccc}
\text { null } & {[0.625,0.75]} & {[0.75,1]} & {[0.25,0.375]} & {[0.25,0.375]} \\
\text { null } & \text { null } & {[0.625,0.75]} & {[0,0.125]} & {[0.25,0.375]} \\
\text { null } & \text { null } & \text { null } & {[0,0.125]} & {[0,0.125]} \\
\text { null } & \text { null } & \text { null } & \text { null } & {[0.625,0.75]} \\
\text { null } & \text { null } & \text { null } & \text { null } & \text { null }
\end{array}\right), \\
& \widetilde{V^{2}}=\left(\begin{array}{ccccc}
\text { null } & 0.5 & {[0,0.375]} & {[0,0.375]} & {[0,0.375]} \\
\text { null } & \text { null } & {[0.25,0.376]} & {[0.25,0.376]} & {[0.25,0.376]} \\
\text { null } & \text { null } & \text { null } & 0.5 & {[0.25,0.376]} \\
\text { null } & \text { null } & \text { null } & \text { null } & {[0.25,0.376]} \\
\text { null } & \text { null } & \text { null } & \text { null } & \text { null }
\end{array}\right) \text {, } \\
& \widetilde{V^{3}}=\left(\begin{array}{ccccc}
\text { null } & {[0.748,0.75]} & {[0,0.249]} & {[0.25,0.499]} & {[0.25,0.499]} \\
\text { null } & \text { null } & {[0,0.249]} & {[0.25,0.499]} & {[0.25,0.499]} \\
\text { null } & \text { null } & \text { null } & {[0.748,0.75]} & {[0.748,0.75]} \\
\text { null } & \text { null } & \text { null } & \text { null } & {[0.25,0.499]} \\
\text { null } & \text { null } & \text { null } & \text { null } & \text { null }
\end{array}\right) \text {, } \\
& \widetilde{V^{4}}=\left(\begin{array}{ccccc}
\text { null } & 0.5 & {[0.249,0.25]} & {[0,0.249]} & {[0,0.249]} \\
\text { null } & \text { null } & {[0.249,0.25]} & {[0.249,0.25]} & {[0.249,0.25]} \\
\text { null } & \text { null } & \text { null } & 0.5 & 0.5 \\
\text { null } & \text { null } & \text { null } & \text { null } & 0.5 \\
\text { null } & \text { null } & \text { null } & \text { null } & \text { null }
\end{array}\right) \text {, }
\end{aligned}
$$




$$
\widetilde{V^{5}}=\left(\begin{array}{ccccc}
\text { null } & \text { null } & \text { null } & \text { null } & \text { null } \\
0.5 & \text { null } & \text { null } & \text { null } & \text { null } \\
{[0.75,0.751]} & {[0.75,0.751]} & \text { null } & \text { null } & \text { null } \\
{[0.751,1]} & {[0.75,0.751]} & 0.5 & \text { null } & \text { null } \\
{[0.751,1]} & {[0.751,1]} & {[0.75,0.751]} & {[0.75,0.751]} & \text { null }
\end{array}\right)
$$

The optimistic consistency index of $\widetilde{V^{k}}, O C I\left(\widetilde{V^{k}}\right)$, and the pessimistic consistency index of $\widetilde{V^{k}}, P C I\left(\widetilde{V^{k}}\right)(k=1,2, \ldots, 5)$, are listed in Table 2 showing high values of consistency according to Remark 5.

\begin{tabular}{llllll}
\multicolumn{5}{c}{ Table 2. Values of $O C I\left(\widetilde{V^{k}}\right)$ and $\operatorname{PCI}\left(\widetilde{V^{k}}\right)(k=1,2, \ldots, 5)$} \\
\hline & $V^{1}$ & $V^{2}$ & $V^{3}$ & $V^{4}$ & $V^{5}$ \\
\hline$P C I\left(\widetilde{V^{k}}\right)$ & 0.825 & 0.825 & 0.816 & 0.933 & 0.883 \\
$O C I\left(\widetilde{V^{k}}\right)$ & 1 & 1 & 1 & 1 & 1 \\
\hline
\end{tabular}

Remark 6. Despite the representation of PIS is a very challenging and complex task in Proposition 1 has been introduced an interval based representation of PIS. This solution is valid but still improvable. It seems relevant for future research to study models that provide fuzzy representations for PIS, but it is not the aim of the current research in this paper.

\section{A CW framework with PIS based on the 2-tuple linguistic model}

his section, a framework for CW dealing with PIS based on 2-tuple linguistic model is proposed.

\subsection{A 2-tuple linguistic framework based on Yager's CW scheme}

This subsection introduces a CW linguistic framework to manage the linguistic information with PIS in real-world problems, which fulfils the phases of the CW scheme showed in Fig. 1, such that it will be able:

- To obtain linguistic inputs

- To represent the personalized individual semantics

- To carry out the CW processes

- Finally, to return linguistic outputs taking into account PIS

The numerical interval individual semantics obtained from the consistencydriven optimization-based model allows reflecting individual differences in 
understanding the meaning of words. Thus, the new CW framework to deal with individual semantics, in which $S=\left\{s_{0}, s_{1}, \ldots, s_{g}\right\}$ is the established linguistic term set, $\Phi=\{A \mid A \subseteq[0,1]\}$ the established numerical domain, and $E=\left\{e_{1}, e_{2}, \ldots, e_{n}\right\}$ the set of $n$ individuals, should extend the scheme of Fig. 1. Therefore, our proposal consists of the scheme depicted in Fig. 2, composed by the following three processes:

- Individual semantics translation. This process translates linguistic terms in $S$ into the individual semantics defined by interval values in the established numerical domain $\Phi$. The individual semantics translation process can be carried out by the consistency-driven optimization-based model introduced in Section 3. Formally, it can be expressed as the mapping $I N S^{k}: S \rightarrow \Phi$, where $I N S^{k}$ is called the individual semantics translation, associated with $e_{k}$.

- Numerical computation. The output of individual semantics translation activates numerical computation over $\Phi$, whose output is an interval numerical value.

- Individual semantics retranslation. It is the inverse operation of individual semantics translation, and it is applied to retranslate the output of numerical computation into linguistic 2-tuples in $\bar{S}$ easy to understand for individuals. The individual semantics retranslation can be expressed as the inverse of $I N S^{k}$, denoted as $I N S^{k,-1}$.

Individual semantics translation

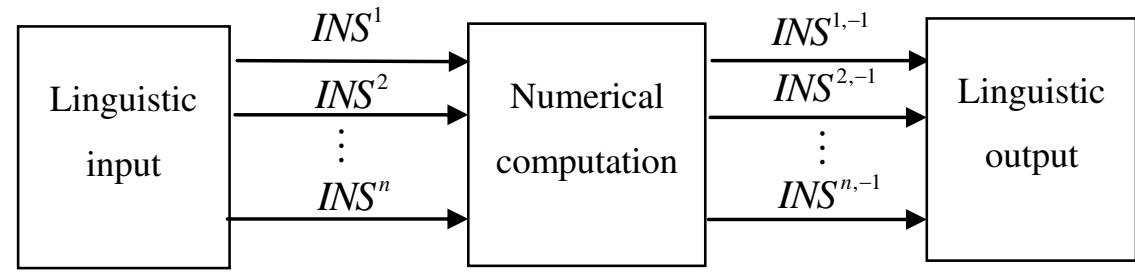

Fig. 2. The framework for the 2-tuple linguistic model with individual semantics

Following, different operators for numerical computation based on the linguistic 2-tuple are further detailed, the other two processes are based on results presented in previous sections and not further detailed here. 


\subsection{Comparison and aggregation: The personalized 2-tuple linguistic opera- tors}

The comparison and aggregation operators in the computational model of the 2-tuple linguistic model have been investigated extensively. However, the existing 2-tuple linguistic models only can be suitable to deal with decision problems in the context that a word has the same numerical meaning for different people.

In this subsection, following the CW framework in Fig. 2, it is proposed the personalized 2-tuple linguistic comparison and aggregation operators for the numerical computation phase to deal with the problem that words mean different things to different people.

Let $S=\left\{s_{0}, s_{1}, \ldots, s_{g}\right\}$ be a linguistic term set, and let $E=\left\{e_{1}, e_{2}, \ldots, e_{m}\right\}$ be the set of decision makers. Let $I N S^{k}$ be the interval numerical scale over $S$, associated with the decision maker $e_{k}$. In the following personalized 2tuple linguistic comparison and aggregation operators are presented.

(1) Personalized 2-tuple linguistic comparison operator

Let $r_{\kappa}$ and $r_{\rho}$ be two linguistic terms provided by decision makers $e_{\kappa}$ and $e_{\rho}$, then

(i) $r_{\kappa} \succ r_{\rho}$ if and only if $I N S^{\kappa}\left(r_{\kappa}\right)>I N S^{\rho}\left(r_{\rho}\right)$;

(ii) $r_{\kappa} \sim r_{\rho}$ if and only if $I N S^{\kappa}\left(r_{\kappa}\right)=I N S^{\rho}\left(r_{\rho}\right)$;

(iii) $r_{\kappa} \prec r_{\rho}$ if and only if $I N S^{\kappa}\left(r_{\kappa}\right)<I N S^{\rho}\left(r_{\rho}\right)$.

Remark 7. There are many proposals for comparing interval values. Without loss of generality, in this paper it is used the comparison operator introduced in [43] to compare interval values.

Example 4. Let $E=\left\{e_{1}, e_{2}, e_{3}\right\}$ be three decision makers, and let $S=$ $\left\{s_{0}, s_{1}, \ldots, s_{4}\right\}$ be the linguistic term set. As shown in Section 3, different decision makers have different interval numerical scales over $S$. Without loss of generality, the individual interval numerical scale $I N S^{k}$ over $S$, associated with $e_{k}$, is set as follows,

$I N S^{1}\left(s_{0}\right)=[0,0.25], I N S^{1}\left(s_{1}\right)=[0.3,0.45], I N S^{1}\left(s_{2}\right)=0.5, I N S^{1}\left(s_{3}\right)=$ $[0.6,0.7]$, and $I N S^{1}\left(s_{4}\right)=[0.75,1]$;

$I N S^{2}\left(s_{0}\right)=0, I N S^{2}\left(s_{1}\right)=[0.1,0.25], I N S^{2}\left(s_{2}\right)=0.5, I N S^{2}\left(s_{3}\right)=$ $[0.8,0.9]$, and $I N S^{2}\left(s_{4}\right)=[0.9,1]$;

$I N S^{3}\left(s_{0}\right)=[0,0.1], I N S^{3}\left(s_{1}\right)=[0.2,0.4], I N S^{3}\left(s_{2}\right)=0.5, I N S^{3}\left(s_{3}\right)=$ $[0.75,0.8]$, and $I N S^{3}\left(s_{4}\right)=[0.8,1]$. 
Let $r_{1}, r_{2}$ and $r_{3}$ be the linguistic terms provided by decision makers $e_{1}$, $e_{2}$ and $e_{3}$, respectively.

If $r_{1}=s_{1}, r_{2}=s_{1}$, and $r_{3}=s_{3}$, using the personalized 2-tuple linguistic comparison operator it can be obtained $r_{2} \prec r_{1} \prec r_{3}$ because of $\operatorname{INS}^{1}\left(r_{1}\right)<$ $I N S^{3}\left(r_{3}\right)<I N S^{2}\left(r_{2}\right)$.

(2) Personalized 2-tuple linguistic aggregation operators

Definition 11. Let $S=\left\{s_{0}, s_{1}, \ldots, s_{g}\right\}, E=\left\{e_{1}, e_{2}, \ldots, e_{m}\right\}$ and $I N S^{k}$ be defined as before. Let $R=\left\{r_{1}, r_{2}, \ldots, r_{m}\right\}$ be a set of linguistic terms to aggregate, where $r_{k} \in S$ are the linguistic terms given by decision makers $e_{k}(k=1,2, \ldots, m)$, and let $W=\left\{w_{1}, w_{2}, \ldots, w_{m}\right\}$ be a weighting vector that satisfies $w_{k} \geq 0$ and $\sum_{k=1}^{m} w_{k}=1$, then the personalized 2-tuple linguistic weighted averaging (PTLWA) operator is defined as

$$
\operatorname{PTLW} A_{W}\left(r_{1}, r_{2}, \ldots, r_{m}\right)=\left(\widetilde{r_{1}}, \widetilde{r_{2}}, \ldots, \widetilde{r_{m}}\right)^{T},
$$

where $\widetilde{r_{k}}=I N S^{k,-1}(q)$ and $q=w_{1} \times I N S^{1}\left(r_{1}\right)+w_{2} \times I N S^{2}\left(r_{2}\right)+\ldots+w_{m} \times$ $I N S^{m}\left(r_{m}\right)$. The personalized 2-tuple linguistic ordered weighted averaging (PTLOWA) operator is computed as

$$
\operatorname{PTLOW} A_{W}\left(r_{1}, r_{2}, \ldots, r_{m}\right)=\left(\widetilde{r_{1}}, \widetilde{r_{2}}, \ldots, \widetilde{r_{m}}\right)^{T},
$$

where $\widetilde{r_{k}}=I N S^{k,-1}(q), q=w_{1} \times I N S^{\sigma(1)}\left(r_{\sigma(1)}\right)+w_{2} \times I N S^{\sigma(2)}\left(r_{\sigma(2)}\right)+$ $\ldots+w_{m} \times I N S^{\sigma(m)}\left(r_{\sigma(m)}\right)$, and $(\sigma(1), \sigma(2), \ldots, \sigma(m))$ is the permutation of $(1,2, \ldots, m)$ such that $I N S^{\sigma(k-1)}\left(r_{\sigma(k-1)}\right) \succ I N S^{\sigma(k)}\left(r_{\sigma(k)}\right)$ for $k=2,3, \ldots, m$.

In Definition 11, $q$ is the numerical computation result over the linguistic terms $\left\{r_{1}, r_{2}, \ldots, r_{m}\right\}$, and $\widetilde{r_{k}}(k=1,2, \ldots, m)$ are the linguistic 2-tuples, which show the different understanding of the decision makers $e_{k}$ to the numerical computation result $q$.

Below, Example 5 illustrates the calculation of the PTLOWA operator. The calculation of the PTLWA operator is similar.

Example 5. Let $E=\left\{e_{1}, e_{2}, e_{3}\right\}, S=\left\{s_{0}, s_{1}, \ldots, s_{4}\right\}$ and $I N S^{k}(k=1,2,3)$ be as Example 4 . Let $r_{1}, r_{2}$, and $r_{3}$ be the linguistic terms provided by decision makers $e_{1}, e_{2}$, and $e_{3}$, respectively. Without loss of generality, let $r_{1}=s_{1}, r_{2}=s_{1}$, and $r_{3}=s_{3}$.

(1) Individual semantics translation. According to $\operatorname{INS}^{k}(k=1,2,3)$ in Example 4, we have $I N S^{1}\left(s_{1}\right)=[0.3,0.45], I N S^{2}\left(s_{1}\right)=[0.1,0.25]$ and $I N S^{3}\left(s_{3}\right)=[0.75,0.8]$. 
(2) Numerical computation. Without loss of generality, let the weighting vector $W=\left\{\frac{1}{3}, \frac{1}{3}, \frac{1}{3}\right\}$, then $q=\frac{1}{3} \times I N S^{1}\left(s_{1}\right)+\frac{1}{3} \times I N S^{2}\left(s_{1}\right)+\frac{1}{3} \times I N S^{3}\left(s_{3}\right)=$ $[0.383,0.5]$.

(3) Individual semantics retranslation. Since $\widetilde{r_{1}}=I N S^{1,-1}(q)=\left(s_{1}, 0.449\right)$,

$\widetilde{r_{2}}=I N S^{2,-1}(q)=\left(s_{2},-0.207\right)$ and $\widetilde{r_{3}}=I N S^{3,-1}(q)=\left(s_{2},-0.345\right)$, we have PTLOW $A_{W}\left(s_{1}, s_{1}, s_{3}\right)=\left(\widetilde{r_{1}}, \widetilde{r_{2}}, \widetilde{r_{3}}\right)^{T}=\left(\left(s_{1}, 0.449\right),\left(s_{2},-0.207\right),\left(s_{2},-0.345\right)\right)^{T}$.

Some desired properties of the PTLOWA operator are introduced. The properties of the PTLWA operator can be analyzed similarly.

Proposition 2. Let $S=\left\{s_{0}, s_{1}, \ldots, s_{g}\right\}, E=\left\{e_{1}, e_{2}, \ldots, e_{m}\right\}, R=\left\{r_{1}, r_{2}, \ldots, r_{m}\right\}$, $W=\left\{w_{1}, w_{2}, \ldots, w_{m}\right\}$, and $I N S^{k}$ be defined as before. Then the PTLOWA operator satisfies the following properties,

(1) Boundary. Let $q_{1}=\min _{\alpha \in\{1, \ldots, m\}} \sum_{k=1}^{m}\left(w_{k} \times I N S^{k}\left(r_{\alpha}\right)\right)$ and $q_{2}=\max _{\alpha \in\{1, \ldots, m\}} \sum_{k=1}^{m}\left(w_{k} \times\right.$ $\left.I N S^{k}\left(r_{\alpha}\right)\right)$. Then $\left(I N S^{1,-1}\left(q_{1}\right), I N S^{2,-1}\left(q_{1}\right), \ldots, I N S^{m,-1}\left(q_{1}\right)\right)^{T} \leq P T L O W A_{W}$ $\left(r_{1}, r_{2}, \ldots, r_{m}\right) \leq\left(I N S^{1,-1}\left(q_{2}\right), I N S^{2,-1}\left(q_{2}\right), \ldots, I N S^{m,-1}\left(q_{2}\right)\right)^{T}$.

(2) Idempotency. PTLOW $A_{W}\left(r_{1}, r_{2}, \ldots, r_{m}\right)=\left(r_{1}, r_{2}, \ldots, r_{m}\right)^{T}$ if $r_{k} \sim r_{t}$ for any $k, t \in\{1,2, \ldots, m\}$.

(3) Commutativity. If $\left(r_{1}^{\prime}, r_{2}^{\prime}, \ldots, r_{m}^{\prime}\right)$ is any permutation of $\left(r_{1}, r_{2}, \ldots, r_{m}\right)$, then we have PTLOW $A_{W}\left(r_{1}^{\prime}, r_{2}^{\prime}, \ldots, r_{m}^{\prime}\right)=P T L O W A_{W}\left(r_{1}, r_{2}, \ldots, r_{m}\right)$.

(4) Monotonicity. PTLOW $A_{W}\left(r_{1}, r_{2}, \ldots, r_{m}\right)>\operatorname{PTLOW} A_{W}\left(r_{1}^{\prime}, r_{2}^{\prime}, \ldots, r_{m}^{\prime}\right)$ if $r_{k} \succ r_{k}^{\prime}$ for $k=1,2, \ldots, m$.

The proof of Proposition 2 is provided in Appendix.

Once it has been introduced different operators for carrying out the $\mathrm{Nu}$ merical Computation process of the CW framework with PIS (see Fig. 2), it is convenient to show the differences between $\mathrm{CW}$ processes carried out by previous models in the literature and our proposal to clarify the differences and advantages of using PIS in those problems in which can be necessary. To do so, below it is proposed a comparison among different functions to compute the numerical indexes according to the linguistic modelling and using the PTLOWA operator.

Let $S, E, W$, and $r_{k}$ be defined as Examples 4 and 5., then consider five different cases:

Case A. The numerical index is computed by the 2-tuple linguistic model [18]:

$I N S^{1}=I N S^{2}=I N S^{3}=\Delta^{-1}$ 
Case B. The numerical index is computed by:

$I N S^{1}=I N S^{2}=I N S^{3}=\{[0,0.25],[0.3,0.45], 0.5,[0.6,0.7],[0.75,1]\}$

Case $\mathrm{C}$. The numerical index is computed by:

$I N S^{1}=I N S^{2}=I N S^{3}=\{0,[0.1,0.25], 0.5,[0.8,0.9],[0.9,1]\}$

Case D. The numerical index is computed by:

$I N S^{1}=I N S^{2}=I N S^{3}=\{[0,0.1],[0.2,0.4], 0.5,[0.75,0.8],[0.8,1]\}$

Case E. The numerical index is computed by using the personalized 2tuple linguistic operators, in which:

$$
\begin{aligned}
& I N S^{1}=\{[0,0.25],[0.3,0.45], 0.5,[0.6,0.7],[0.75,1]\}, \\
& I N S^{2}=\{0,[0.1,0.25], 0.5,[0.8,0.9],[0.9,1]\}, \\
& I N S^{3}=\{[0,0.1],[0.2,0.4], 0.5,[0.75,0.8],[0.8,1]\} .
\end{aligned}
$$

Comparing the results obtained, among the numerical indexes in different 2-tuple linguistic modelling showed in Table 3, can be found out that the personalized 2-tuple linguistic operators provide not only obvious different results because of computations but also different rankings due to the consideration of different meaning of linguistic information by each expert.

Table 3. Results for different numerical indexes

\begin{tabular}{llc}
\hline & Comparison & Weighted averaging operator \\
\hline Case A & $r_{1} \sim r_{2} \prec r_{3}$ & $\left(s_{2},-0.333\right)$ \\
Case B & $r_{1} \sim r_{2} \prec r_{3}$ & $\left(s_{2},-0.432\right)$ \\
Case C & $r_{1} \sim r_{2} \prec r_{3}$ & $\left(s_{2},-0.337\right)$ \\
Case D & $r_{1} \sim r_{2} \prec r_{3}$ & $\left(s_{2},-0.317\right)$ \\
Case E & $r_{3} \succ r_{1} \succ r_{2}$ & $\left(\left(s_{1}, 0.449\right),\left(s_{2},-0.21\right),\left(s_{2},-0.351\right)\right)^{T}$ \\
\hline
\end{tabular}

\section{Solving a linguistic GDM problem with PIS: A consensus based model}

This section presents the application of the PIS model to deal with the consensus-based linguistic GDM with individual semantics. Specifically, it is introduced the notation for GDM problems with individual semantics together a resolution framework and finally a consensus reaching process is provided and developed.

\subsection{A GDM framework with PIS}

Let $S=\left\{s_{0}, s_{1}, \ldots, s_{g}\right\}$ be a linguistic term set, $X=\left\{X_{1}, X_{2}, \ldots, X_{n}\right\}$ be a set of alternatives, and $E=\left\{e_{1}, e_{2}, \ldots, e_{m}\right\}$ be a set of decision makers. In the GDM with individual semantics, each decision maker provides his/her 
preferences over $X$ by a linguistic preference relation $L^{k}=\left(l_{i j}^{k}\right)_{n \times n}(k=$ $1,2, \ldots, m)$, where $l_{i j}^{k} \in S$ estimates the preference degree of decision maker $e_{k}$ for alternative $X_{i}$ over $X_{j}$. Meanwhile, decision makers have their individual semantics over $S$, namely, they use different interval numerical scales of $S$. Consequently, it is necessary to support decision makers, who have individual semantics described by individual interval numerical scales $I N S^{k}$ over $S$, to reach an agreed solution for the linguistic GDM problem.

Therefore, a new framework to deal with the consensus-based linguistic GDM with individual semantics is introduced. It includes three processes depicted in Fig. 3: individual semantics translation process, selection process and consensus reaching process.

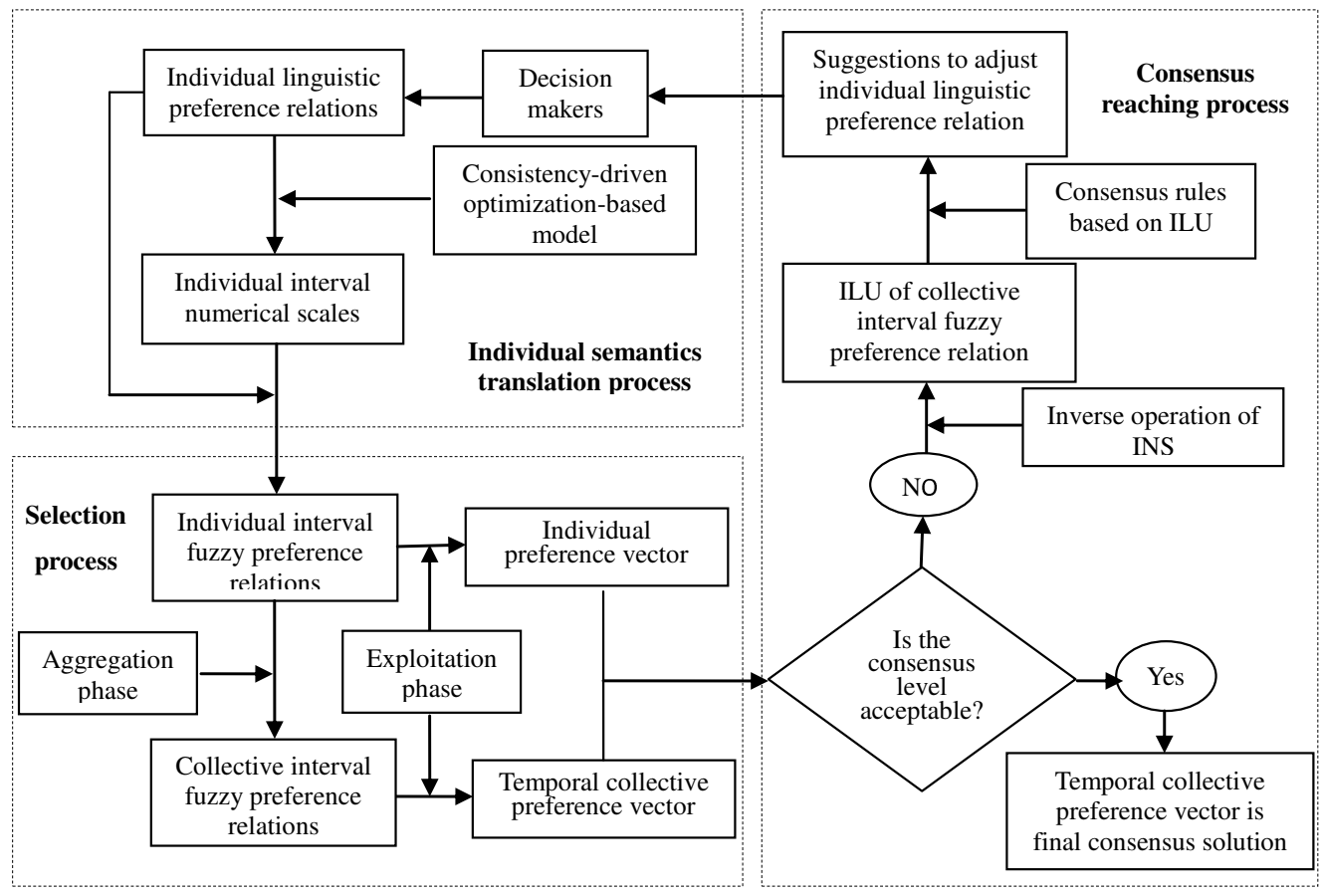

Fig. 3. The framework for the consensus-based linguistic GDM with PIS

(1) Individual semantics translation process

The individual semantics translation process uses the consistency-driven optimization-based model proposed in Section 3.2 to generate the individual interval numerical scales of $S, I N S^{k}$, by applying the individual linguistic 
preference relation $L^{k}$ as data resource. Using the individual interval numerical scale $I N S^{k}$ to quantify $L^{k}$ obtains the individual interval fuzzy preference relation $\left.\widetilde{V^{k}}=\widetilde{\left(v_{i j}^{k}\right.}\right)_{n \times n}$, where

$$
\widetilde{v_{i j}^{k}}=\left[v_{i j}^{k-}, v_{i j}^{k+}\right]=I N S^{k}\left(l_{i j}^{k}\right)(k=1,2, \ldots, m) .
$$

\section{(2) Selection process}

It aims at obtaining the collective ranking of alternatives by applying two phases: aggregation phase and exploitation phase.

The aggregation phase aggregates individual interval fuzzy preference relations $\left\{\widetilde{V^{1}}, \widetilde{V^{2}}, \ldots, \widehat{V^{m}}\right\}$ into a collective preference relation $\widetilde{V^{c}}=\left(\widetilde{v_{i j}^{c}}\right)_{n \times n}$. The aggregation operation can be carried out by means of either the weighted average (WA) operator or ordered weighted average (OWA) operator [47]. In this paper, the WA operator is used, i.e.,

$$
\widetilde{v_{i j}^{c}}=\left[v_{i j}^{c-}, v_{i j}^{c+}\right]=\left[\sum_{k=1}^{m} \lambda_{k} \cdot v_{i j}^{k-}, \sum_{k=1}^{m} \lambda_{k} \cdot v_{i j}^{k+}\right],
$$

where $\lambda=\left\{\lambda_{1}, \lambda_{2}, \ldots, \lambda_{m}\right\}$ is the weighting vector of decision makers $\left\{e_{1}, e_{2}, \ldots, e_{m}\right\}$ that satisfies $\lambda_{k} \in[0,1]$ and $\sum_{k=1}^{m} \lambda_{k}=1$.

In the exploitation phase, the collective preference vector $Z^{c}=\left(z_{1}^{c}, z_{2}^{c}, \ldots, z_{n}^{c}\right)^{T}$ is obtained from $\widetilde{V^{c}}$ to order alternatives, where

$$
z_{i}^{c}=\left[\sum_{j=1}^{n} w_{j} \cdot v_{i j}^{c-}, \sum_{j=1}^{n} w_{j} \cdot v_{i j}^{c+}\right],
$$

and $W=\left\{w_{1}, w_{2}, \ldots, w_{n}\right\}$ is an associated weighting vector that satisfies $w_{j} \in[0,1]$ and $\sum_{j=1}^{n} w_{j}=1$.

From the values $z_{i}^{c}$, the ranking of alternatives $\left\{X_{1}, X_{2}, \ldots, X_{n}\right\}$ is obtained. The larger the value of $z_{i}^{c}$, the better the alternative.

(3) Consensus reaching process

It aims at reaching a higher agreement level among decision makers. The details of the consensus reaching process are introduced in Section 5.2. 


\subsection{Consensus reaching process}

A consensus reaching process can be viewed as an iterative process with several consensus rounds, in which the decision makers adjust their preferences following the consensus rules until the maximum possible consensus level is achieved. Generally consensus reaching process includes two parts [34]: (i) A consensus measure process computes the level of agreement among experts and, (ii) A feedback mechanism guides the process to improve the agreement among them.

(1) Consensus measure

Let $Z^{k}=\left(z_{1}^{k}, z_{2}^{k}, \ldots, z_{n}^{k}\right)^{T}$ be the individual preference vector obtained from $\widetilde{V^{k}}$ to rank alternatives, where

$$
z_{i}^{k}=\left[\sum_{j=1}^{n} w_{j} \cdot v_{i j}^{k-}, \sum_{j=1}^{n} w_{j} \cdot v_{i j}^{k+}\right], \text { for } i=1,2, \ldots, n .
$$

Let $O^{c}=\left(o_{1}^{c}, o_{2}^{c}, \ldots, o_{n}^{c}\right)^{T}$, where $o_{i}^{c}$ is the position of alternative $X_{i}$ in $Z^{c}$. For example, if $Z^{c}=([0.1,0.2],[0.6,0.7],[0.3,0.4],[0.8,0.9])$, then $O^{c}=$ $(4,2,3,1)^{T}$. Similarly, we get $O^{k}=\left(o_{1}^{k}, o_{2}^{k}, \ldots, o_{n}^{k}\right)^{T}$, where $o_{i}^{k}$ is the position of alternative $X_{i}$ in $Z^{k}$.

The consensus measure used in our proposal for consensus reaching process is defined as:

Definition 12. The consensus level associated with decision maker $e_{k}, C L_{k} \in$ $[0,1]$, is given by

$$
C L_{k}=1-2 \sum_{i=1}^{n} \frac{\left|o_{i}^{c}-o_{i}^{k}\right|}{n^{2}} .
$$

The consensus level of all decision makers $\left\{e_{1}, e_{2}, \ldots, e_{m}\right\}, C L \in[0,1]$, is given by

$$
C L=1-2 \sum_{k=1}^{m} \sum_{i=1}^{n} \frac{\left|o_{i}^{c}-o_{i}^{k}\right|}{m n^{2}} .
$$

A larger $C L$ value indicates a higher consensus degree among the decision makers $\left\{e_{1}, e_{2}, \ldots, e_{m}\right\}$.

(2) Feedback mechanism

In our proposal the feedback mechanism is based on different consensus rules that help decision makers to make their opinions closer across the 
consensus reaching process. Before introducing the consensus rules, it is proposed the concept of the individual linguistic understanding of the collective interval fuzzy preference relation, associated with each decision maker (see Definition 13), which provides the basis of the consensus rules.

Definition 13. Let $I N S^{k}$ be an ordered interval numerical scale on $S$, associated with the decision maker $e_{k}$, and let $\widetilde{V^{c}}=\left(\widetilde{v_{i j}^{c}}\right)_{n \times n}$ be a collective interval fuzzy preference relation. Then $L^{k *}=\left(l_{i j}^{k *}\right)_{n \times n}$, where

$$
l_{i j}^{k *}=I N S^{k,-1}\left(\widetilde{v_{i j}^{c}}\right),
$$

is called the individual linguistic understanding of the collective interval fuzzy preference relation $\widetilde{V^{c}}$, associated with the decision maker $e_{k}$.

The individual linguistic understanding reflects the linguistic meaning of the collective interval fuzzy preference relation $\widetilde{V^{c}}$, associated with individual decision makers. According to Eqs. (33), (34) and (39), the individual linguistic understanding of $\widetilde{V^{c}}$ can be expressed by a PTLWA operator, i.e.,

$$
\left(l_{i j}^{1 *}, l_{i j}^{2 *}, \ldots, l_{i j}^{m *}\right)=\operatorname{PTLW} A_{\lambda}\left(l_{i j}^{1}, l_{i j}^{2}, \ldots, l_{i j}^{m}\right) .
$$

Naturally, different decision makers have different linguistic understanding over $\widetilde{V^{c}}$. The individual linguistic understanding of $\widetilde{V^{c}}$ is illustrated in Example 6 .

Example 6. Let $E=\left\{e_{1}, e_{2}\right\}$ and let $S=\left\{s_{0}, s_{1}, \ldots, s_{4}\right\}$. According to Section 3, different decision makers set different interval numerical scales over $S$. Without loss of generality, the individual interval numerical scales $I N S^{1}$ and $I N S^{2}$ over $S$, associated with $e_{1}$ and $e_{2}$, respectively, is defined as follows: $I N S^{1}\left(s_{0}\right)=[0,0.25], I N S^{1}\left(s_{1}\right)=[0.3,0.45], I N S^{1}\left(s_{2}\right)=0.5, I N S^{1}\left(s_{3}\right)=$ $[0.6,0.7]$ and $I N S^{1}\left(s_{4}\right)=[0.75,1]$.

$I N S^{2}\left(s_{0}\right)=0, I N S^{2}\left(s_{1}\right)=[0.1,0.25], I N S^{2}\left(s_{2}\right)=0.5, I N S^{2}\left(s_{3}\right)=[0.8,0.9]$ and $I N S^{2}\left(s_{4}\right)=[0.9,1]$.

Let the collective interval fuzzy preference relation $\widetilde{V^{c}}$ be as follows,

$$
\widetilde{V^{c}}=\left(\begin{array}{cccc}
\text { null } & {[0.3,0.4]} & {[0.4,0.6]} & {[0.75,1]} \\
\text { null } & \text { null } & {[0.2,0.6]} & {[0,0.3]} \\
\text { null } & \text { null } & \text { null } & {[0.7,0.8]} \\
\text { null } & \text { null } & \text { null } & \text { null }
\end{array}\right)
$$


Then, the individual linguistic understandings of $\widetilde{V^{c}}$, associated with decision makers $e_{1}$ and $e_{2}$, are $L^{1 *}$ and $L^{2 *}$, respectively, i.e.,

$$
L^{1 *}=\left(\begin{array}{cccc}
\text { null } & \left(s_{1},-0.25\right) & \left(s_{1},-0.235\right) & s_{4} \\
\text { null } & \text { null } & \left(s_{2},-0.4\right) & \left(s_{0}, 0.46\right) \\
\text { null } & \text { null } & \text { null } & \left(s_{4},-0.333\right) \\
\text { null } & \text { null } & \text { null } & \text { null }
\end{array}\right) \text {, }
$$

and

$$
L^{2 *}=\left(\begin{array}{cccc}
\text { null } & \left(s_{2},-0.472\right) & \left(s_{2},-0.345\right) & \left(s_{3}, 0.25\right) \\
\text { null } & \text { null } & \left(s_{2}, 0.16\right) & \left(s_{1}, 0.18\right) \\
\text { null } & \text { null } & \text { null } & \left(s_{3},-0.12\right) \\
\text { null } & \text { null } & \text { null } & \text { null }
\end{array}\right)
$$

According to $L^{1 *}$ and $L^{2 *}$, decision makers $e_{1}$ and $e_{2}$ have different linguistic understanding over $\widetilde{V^{c}}$. Based on the individual linguistic understanding of the collective interval fuzzy preference relation and the consensus level $C L_{k}$ associated with $e_{k}$, two consensus rules namely, identification rule and direction rule to guide the feedback process are introduced:

(1) Identification rule. The identification rule identifies the decision makers contributing less to reach a high degree of consensus.

From the ranking position of each decision maker $e_{k}$ according to $C L_{k}$, the larger the $C L_{k}$, the higher position of decision maker $e_{k}$. If the decision maker's position is high, then the decision maker does not need to change his/her preferences, but if it is low then the decision maker has to change his/her preferences.A satisfaction consensus threshold $\overline{C L}$ is computed to calculate how many decision makers need to change their preferences. If $C L_{k}<\overline{C L}, \overline{C L} \in[0,1]$, the decision maker $e_{k}$ needs to change his/her preferences. Generally, the decision maker $e_{\tau}$, whose consensus level $C L_{\tau}=$ $\min _{k} C L_{k}(k=1,2, \ldots, m)$, needs to change his/her preferences.

(2) Direction rule. The direction rule finds out the direction to change the preferences of decision makers.

Let $L^{k *}=\left(l_{i j}^{k *}\right)_{n \times n}$ be the individual linguistic understanding of the collective interval fuzzy preference relation $\widetilde{V^{c}}$, associated with $e_{k}$. Let $\overline{L^{k}}=\left(\overline{l_{i j}^{k}}\right)_{n \times n}$ be the adjusted linguistic preference relation associated with $e_{k}$. Then the direction rules are as follows:

(i) If $l_{i j}^{k}$ is smaller than $l_{i j}^{k *}$, then decision maker $e_{k}$ should increase the evaluation associated with the pairwise $\left(X_{i}, X_{j}\right)$. Specifically, the adjusted 
preference value should be $\overline{l_{i j}^{k}} \in\left\{s \mid s \in S, s \in\left(l_{i j}^{k}, l_{i j}^{k *}\right]\right\}$.

(ii) If $l_{i j}^{k}=l_{i j}^{k *}$, then the decision maker $e_{k}$ should not change the evaluations associated with the pairwise $\left(X_{i}, X_{j}\right)$.

(iii) If $l_{i j}^{k}$ is larger than $l_{i j}^{k *}$, then the decision maker $e_{k}$ should decrease the evaluation associated with the pairwise $\left(X_{i}, X_{j}\right)$. Specifically, the adjusted preference value should be $\overline{l_{i j}^{k}} \in\left\{s \mid s \in S, s \in\left[l_{i j}^{k *}, l_{i j}^{k}\right)\right\}$.

The following Algorithm 1 provides a formal description of the consensus reaching process.

\section{Algorithm 1}

Input: The individual linguistic preference relation based on $S, L^{k}=$ $\left(l_{i j}^{k}\right)_{n \times n}(k=1,2, \ldots, m)$, the weighting vectors $\lambda=\left\{\lambda_{1}, \lambda_{2}, \ldots, \lambda_{m}\right\}$ and $W=$ $\left\{w_{1}, w_{2}, \ldots, w_{n}\right\}$, the established consensus threshold $\overline{C L}$, and the established maximum number of iterations $h_{\max }$.

Output: Adjusted linguistic preference relation $\overline{L^{k}}=\left(\overline{l_{i j}^{k}}\right)_{n \times n}(k=1,2, \ldots, m)$.

Step 1: Let $h=0$ and $L_{h}^{k}=\left(l_{i j, h}^{k}\right)_{n \times n}=\left(l_{i j}^{k}\right)_{n \times n}(k=1,2, \ldots, m)$

Step 2: The consistency-driven optimization-based model presented in Section 3.2 is used to set the individual interval numerical scales $I N S^{k}$.

Step 3: Using $I N S^{k}$ to quantify $L_{h}^{k}$ obtains the individual interval fuzzy preference relation $\widetilde{V_{h}^{k}}$. Then, using Eq. (34) obtains the collective interval fuzzy preference relation $\widetilde{V_{h}^{c}}$. Next, using Eqs. (35) and (36) obtains the collective preference vector $Z^{c}=\left(z_{1}^{c}, z_{2}^{c}, \ldots, z_{n}^{c}\right)^{T}$ and the individual preference vector $Z^{k}=\left(z_{1}^{k}, z_{2}^{k}, \ldots, z_{n}^{k}\right)^{T}$. Finally, based on Definition 12, the consensus level $C L_{h}$ is calculated. If $C L_{h}>\overline{C L}$ or $h>h_{\max }$, then go to Step 6; otherwise, continue with the next step.

Step 4: Using Eq. (39) obtains the individual linguistic understanding of the collective interval fuzzy preference relation $\widetilde{V_{h}^{c}}$, associated with $e_{k}$, $L_{h}^{k *}=\left(l_{i j, h}^{k *}\right)_{n \times n}(k=1,2, \ldots, m)$.

Step 5: Based on the identification rule, the decision maker $e_{\tau}$, who has the lowest consensus level, needs to change his/her preferences. Then, according to the direction rule, the adjusted suggestions associated with decision maker $e_{\tau}$ and the pairwise $\left(X_{i}, X_{j}\right)$ are obtained, i.e.,

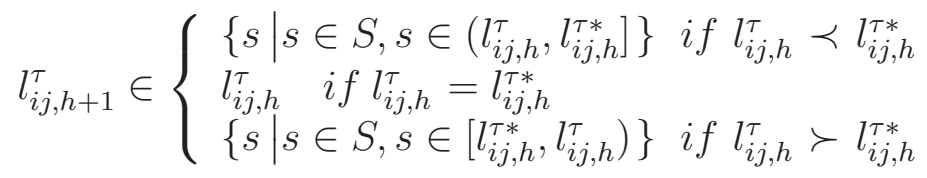

Based on Eq. (41), construct the new individual linguistic preference relation $L_{h+1}^{\tau}=\left(l_{i j, h+1}^{\tau}\right)_{n \times n}$. Let $h=h+1$. Then, go to Step 2 . 
Step 6: Let $\overline{L^{k}}=L_{h}^{k}$. Output the adjusted linguistic preference relation $\overline{L^{k}}=\left(\overline{l_{i j}^{k}}\right)_{n \times n}(k=1,2, \ldots, m)$.

Next, we provide Example 7 to illustrate the selection process and the consensus reaching process.

Example 7. Once finished the individual semantics translation process in Example 4, we keep solving the problem (Example 3) to apply the selection process and the consensus reaching process to it.

\section{(1) Selection process}

Without loss of generality, let the weighting vectors $\lambda=W=\left\{\frac{1}{5}, \frac{1}{5}, \frac{1}{5}, \frac{1}{5}, \frac{1}{5}\right\}$. Using Eq. (34) obtains the collective interval fuzzy preference relation, $\widetilde{V^{c}}=\left(\widetilde{v_{i j}^{c}}\right)_{5 \times 5}$, i.e.,

$$
\widetilde{v_{i j}^{c}}=\left[v_{i j}^{c-}, v_{i j}^{c+}\right]=\left[(1 / 5) \sum_{k=1}^{5} v_{i j}^{k-},(1 / 5) \sum_{k=1}^{5} v_{i j}^{k+}\right] .
$$

Matrix $\widetilde{V^{c}}$ is listed as follows,

$$
\widetilde{V^{c}}=\left(\begin{array}{ccccc}
{[0.5,0.5]} & {[0.575,0.6]} & {[0.25,0.425]} & {[0.1,0.349]} & {[0.1,0.349]} \\
{[0.4,0.425]} & {[0.5,0.5]} & {[0.275,0.375]} & {[0.2,0.3]} & {[0.2,0.35]} \\
{[0.575,0.75]} & {[0.625,0.725]} & {[0.5,0.5]} & {[0.45,0.475]} & {[0.349,0.4]} \\
{[0.651,0.9]} & {[0.7,0.8]} & {[0.525,0.55]} & {[0.5,0.5]} & {[0.375,0.475]} \\
{[0.651,0.9]} & {[0.65,0.85]} & {[0.6,0.651]} & {[0.525,0.625]} & {[0.5,0.5]}
\end{array}\right) .
$$

Then using Eq. (35) yields $z_{i}^{c}=\left[(1 / 5) \sum_{j=1}^{5} v_{i j}^{c-},(1 / 5) \sum_{j=1}^{5} v_{i j}^{c+}\right]$. The values of $z_{i}^{c}(i=1,2, \ldots, 5)$ are listed below, $z_{1}^{c}=[0.305,0.445], z_{2}^{c}=[0.315,0.39], z_{3}^{c}=[0.5,0.57], z_{4}^{c}=[0.55,0.645]$, and $z_{5}^{c}=[0.585,0.705]$.

The larger the value of $z_{i}^{c}$, the better the alternative. Based on the comparison operations of interval numbers [43], the collective ranking of alternatives is $X_{5} \succ X_{4} \succ X_{3} \succ X_{1} \succ X_{2}$.

Similarly, we can get the individual rankings of alternatives, they are as follows,

$$
\begin{aligned}
& e_{1}: X_{4} \succ X_{5} \succ X_{1} \succ X_{2} \succ X_{3} \\
& e_{2}: X_{5} \succ X_{4} \sim X_{3} \succ X_{2} \succ X_{1} \\
& e_{3}: X_{3} \succ X_{5} \succ X_{4} \succ X_{1} \succ X_{2} \\
& e_{4}: X_{5} \succ X_{4} \succ X_{3} \succ X_{2} \succ X_{1}
\end{aligned}
$$


$e_{5}: X_{5} \succ X_{4} \sim X_{3} \succ X_{2} \succ X_{1}$

(2) Consensus reaching process

According to Eq. (37), $C L_{1}=0.52, C L_{2}=0.76, C L_{3}=0.68, C L_{4}=$ 0.84, and $C L_{5}=0.76$. Then, based on Eq. (38), the consensus level of all decision makers is $C L=0.712$.

The consensus rules are then applied to help decision makers reach a high consensus. The consensus rules are carried out in the following two steps:

(i) Identification rule

From the position ranking of each decision maker $e_{k}$ according to $C L_{k}$, it is found that the position of the decision maker $e_{1}$ in the ranking is the lowest. Clearly, the decision maker $e_{1}$ needs to change his/her preferences.

(ii) Direction rule

Firstly, the individual linguistic understanding of collective interval fuzzy preference relation $\widetilde{V^{c}}$, associated with the decision maker $e_{1}, L^{1 *}$, is obtained:

$$
L^{1 *}=\left(\begin{array}{ccccc}
\text { null } & \left(s_{2}, 0.01\right) & \left(s_{1}, 0.08\right) & \left(s_{1},-0.352\right) & \left(s_{1},-0.352\right) \\
\text { null } & \text { null } & \left(s_{1}, 0.08\right) & \left(s_{1},-0.25\right) & \left(s_{1},-0.15\right) \\
\text { null } & \text { null } & \text { null } & \left(s_{1},-0.2\right) & \left(s_{1}, 0.357\right) \\
\text { null } & \text { null } & \text { null } & \text { null } & \left(s_{2},-0.44\right) \\
\text { null } & \text { null } & \text { null } & \text { null } & \text { null }
\end{array}\right) .
$$

Then, let the decision maker $e_{1}$ change his/her preference values according to the direction rule, the new preference relation $L^{1}$ is obtained as follows:

$$
L^{1}=\left(\begin{array}{ccccc}
\text { null } & s_{2} & s_{1} & s_{0} & s_{1} \\
\text { null } & \text { null } & s_{1} & s_{0} & s_{1} \\
\text { null } & \text { null } & \text { null } & s_{1} & s_{1} \\
\text { null } & \text { null } & \text { null } & \text { null } & s_{3} \\
\text { null } & \text { null } & \text { null } & \text { null } & \text { null }
\end{array}\right)
$$

Applying the selection process again,the individual ranking of alternatives is obtained, associated with decision maker $e_{1}$, that is $X_{4} \succ X_{5} \succ X_{3} \succ X_{1} \sim$ $X_{2}$, and the collective ranking of alternatives, $X_{5} \succ X_{4} \succ X_{3} \succ X_{2} \succ X_{1}$. Then, applying the consensus reaching process again, the consensus level of all decision makers is obtained: $C L=0.824$.

\section{Conclusions}

In this paper it has been introduced a Personalized Individual Semantics (PIS) approach to model and solve linguistic GDM problems with prefer- 
ence relations to improve the management of different meanings of words for different people.

First a consistency-driven optimization-based model to personalize and represent the individual semantics based on the interval numerical scale is introduced. Second a new CW framework based on the 2-tuple linguistic model for dealing with personalized individual semantics is developed and eventually both are applied to linguistic GDM problem with a consensus reaching process.

In the future, we plan to work on the potential use of PIS for large scale decision making $[24,25,26,35,37,44]$ to handle large groups with different PIS according to their preferences.

\section{Acknowledgments}

Yucheng Dong would like to acknowledge the financial support of grants (Nos. 71171160, 71571124) from NSF of China, and a grant (No.xq15b01) from SSEM key research center at Sichuan province. Enrique Herrera-Viedma and Luis Martínez would like to acknowledge the FEDER funds under Grant TIN2013-40658-P and TIN2015-66524-P respectively.

\section{References}

\section{References}

[1] M.A. Abchir and I. Truck. Towards an extension of the 2-tuple linguistic model to deal with unbalanced linguistic term sets. Kybernetika, 49(1):164-180, 2013.

[2] S. Alonso, F. Chiclana, F. Herrera, E. Herrera-Viedma, J. Alcalá-Fdez, and C. Porcel. A consistency-based procedure to estimate missing pairwise preference values. International Journal of Intelligent Systems, 23(2):155-175, 2008.

[3] H. Bustince, F. Herrera, and J. Montero. Fuzzy Sets and Their Extensions: Representation, Aggregation and Models:Intelligent Systems from Decision Making to Data Mining, Web Intelligence and Computer Vision, volume 220 of Studies in Fuzziness and Soft Computing. SpringerVerlag, 2008. 
[4] F. Chiclana, E. Herrera-Viedma, S. Alonso, and F. Herrera. Cardinal consistency of reciprocal preference relations: A characterization of multiplicative transitivity. IEEE Transactions on Fuzzy Systems, 17(1):1423, Feb 2009.

[5] Y. Dong, X. Chen, C.C. Li, W.C. Hong, and Y.F. Xu. Consistency issues of interval pairwise comparison matrices. Soft Computing, 19(8):2321$2335,2015$.

[6] Y. Dong and E. Herrera-Viedma. Consistency-driven automatic methodology to set interval numerical scales of 2-tuple linguistic term sets and its use in the linguistic GDM with preference relation. IEEE Transactions on Cybernetics, 45(4):780-792, 2015.

[7] Y. Dong, C.C. Li, and F. Herrera. Connecting the numerical scale model to the unbalanced linguistic term sets. In IEEE International Conference on Fuzzy Systems (FUZZ-IEEE), pages 455-462, 2014.

[8] Y. Dong, C.C. Li, and F. Herrera. An optimization-based approach to adjusting unbalanced linguistic preference relations to obtain a required consistency level. Information Sciences, 292:27-38, 2015.

[9] Y. Dong, C.C. Li, Y.F. Xu, and X. Gu. Consensus-based group decision making under multi-granular unbalanced 2-tuple linguistic preference relations. Group Decision and Negotiation, 24(2):217-242, 2015.

[10] Y. Dong, Y. Xu, and H. Li. On consistency measures of linguistic preference relations. European Journal of Operational Research, 189(2):430444, 2008.

[11] Y. Dong, Y. Xu, and S. Yu. Computing the numerical scale of the linguistic term set for the 2-tuple fuzzy linguistic representation model. IEEE Transactions on Fuzzy Systems, 17(6):1366-1378, 2009.

[12] Y. Dong, G. Zhang, W.C. Hong, and S. Yu. Linguistic computational model based on 2-tuples and intervals. IEEE Transactions on Fuzzy Systems, 21(6):1006-1018, 2013.

[13] H. Doukas, C. Karakosta, and J. Psarras. Computing with words to assess the sustainability of renewable energy options. Experts Systems with Applications, 37(7):5491-5497, 2010. 
[14] M. Espinilla, J. Liu, and L. Martínez. An extended hierarchical linguistic model for decision-making problems. Computational Intelligence, 27(3):489-512, 2011.

[15] F. Herrera, S. Alonso, F. Chiclana, and E. Herrera-Viedma. Computing with words in decision making: Foundations, trends and prospects. Fuzzy Optimization and Decision Making, 8(4):337-364, 2009.

[16] F. Herrera, E. Herrera-Viedma, and L. Martínez. A fusion approach for managing multi-granularity linguistic terms sets in decision making. Fuzzy Sets and Systems, 114(1):43-58, 2000.

[17] F. Herrera, E. Herrera-Viedma, and L. Martínez. A fuzzy linguistic methodology to deal with unbalanced linguistic term sets. IEEE Transactions on Fuzzy Systems, 16(2):354-370, 2008.

[18] F. Herrera and L. Martínez. A 2-tuple fuzzy linguistic representation model for computing with words. IEEE Transactions on Fuzzy Systems, $8(6): 746-752,2000$.

[19] F. Herrera and L. Martínez. A model based on linguistic 2-tuples for dealing with multigranular hierarchical linguistic context in multi-expert decision making. IEEE Transactions on Systems, Man and Cybernetics, Part B: Cybernetics, 31(2):227-234, 2001.

[20] E. Herrera-Viedma, F.J. Cabrerizo, J. Kacprzyk, and W. Pedrycz. A review of soft consensus models in a fuzzy environment. Information Fusion, 17(4):4-13, 2014.

[21] E. Herrera-Viedma, F. Chiclana, F. Herrera, and S. Alonso. Group decision-making model with incomplete fuzzy preference relations based on additive consistency. IEEE Transactions on Systems, Man and Cybernetics, Part B, Cybernetics, 37(1):176-189, 2007.

[22] E. Herrera-Viedma, F. Herrera, F. Chiclana, and M. Luque. Some issues on consistency of fuzzy preference relations. European Journal of Operational Research, 154(1):98-109, 2004.

[23] C-C. Li and Y. Dong. Multi-attribute group decision making methods with proportional 2-tuple linguistic assessments and weights. International Journal of Computational Intelligence Systems, 7(4):758-770, 2014. 
[24] B. Liu, Y. Shen, X. Chen, Y. Chen, and X. Wang. A partial binary tree dea-da cyclic classification model for decision makers in complex multi-attribute large-group interval-valued intuitionistic fuzzy decisionmaking problems. Information Fusion, 18(3):119-130, 2014.

[25] B. Liu, Y. Shen, Y. Chen, X. Chen, and Y. Wang. A two-layer weight determination method for complex multi-attribute large-group decision-making experts in a linguistic environment. Information $\mathrm{Fu}$ sion, 23:156-165, 2015.

[26] Y. Liu, Z.P. Fan, and X. Zhang. A method for large group decisionmaking based on evaluation information provided by participators from multiple groups. Information Fusion, in press, 2015.

[27] L. Martínez and F. Herrera. An overview on the 2-tuple linguistic model for computing with words in decision making: Extensions, applications and challenges. Information Sciences, 207(1):1-18, 2012.

[28] L. Martínez, D. Ruan, and F. Herrera. Computing with words in decision support systems: An overview on models and applications. International Journal of Computational Intelligence Systems, 3(4):382-395, 2010.

[29] J.M. Mendel and D. Wu. Perceptual Computing: Aiding People in Making Subjective Judgments. IEEE-Wiley, 2010.

[30] J.M. Mendel, L.A. Zadeh, E. Trillas, R.R. Yager, J. Lawry, H. Hagras, and S. Guadarrama. What computing with words means to me: Discussion forum. IEEE Computational Intelligence Magazine, 5(1):20-26, 2010 .

[31] J.M. Merigó, M. Casanovas, and L. Martínez. Linguistic aggregation operators for linguistic decision making based on the dempster-shafer theory of evidence. International Journal of Uncertainty, Fuzziness and Knowledge-Based Systems, 18(3):287-304, 2010.

[32] G.A. Miller. The magical number seven plus or minus two: Some limits on our capacity for processing information. Psychological Review, 63:8197, 1956.

[33] J.A. Morente-Molinera, I.J. Pérez, M.R. Ureña, and E. Herrera-Viedma. On multi-granular fuzzy linguistic modelling in group decision making 
problems: a systematic review and future trends. Knowledge-Based Systems, 74:49-60, 2015.

[34] I. Palomares, F.J. Estrella, L. Martínez, and F. Herrera. Consensus under a fuzzy context: Taxonomy, analysis framework AFRYCA and experimental case of study. Information Fusion, 20:252-271, 2014.

[35] I. Palomares, L. Martínez, and F. Herrera. A consensus model to detect and manage noncooperative behaviors in large-scale group decision making. IEEE Transactions on fuzzy systems, 22(3):516-530, 2014.

[36] W. Pedrycz, P. Ekel, and R. Parreiras. Fuzzy Multicriteria DecisionMaking: Models, Methods and Applications. John Wiley \& Sons, Ltd. Chichester, UK, 2010.

[37] F.J. Quesada, I. Palomares, and L. Martínez. Managing experts behavior in large-scale consensus reaching processes with uninorm aggregation operators. Applied Soft Computing, 35:873-887, 2015.

[38] R.M. Rodríguez and L. Martínez. An analysis of symbolic linguistic computing models in decision making. International Journal of General Systems, 42(1):121-136, 2013.

[39] K.S. Schmucker. Fuzzy Sets, Natural Language Computations, and Risk Analysis. Computer Science Press, Rockville, MD, 1984.

[40] M. Tong and P.P. Bonissone. A linguistic approach to decision making with fuzzy sets. IEEE Transactions on Systems, Man and Cybernetics, 10(11):716-723, 1980.

[41] J.H. Wang and J. Hao. A new version of 2-tuple fuzzy linguistic representation model for computing with words. IEEE Transactions on Fuzzy Systems, 14(3):435-445, 2006.

[42] Y.M. Wang, J.B. Yang, and D.L. Xu. Interval weight generation approaches based on consistency test and interval comparison matrices. Applied Mathematics and Computation, 167(1):252-273, 2005.

[43] Y.M. Wang, J.B. Yang, and D.L. Xu. A preference aggregation method through the estimation of utility intervals. Computers and Operations Research, 32:2027-2049, 2005. 
[44] X.H. Xu, Z.J. Du, and X.H. Chen. Consensus model for multi-criteria large-group emergency decision making considering non-cooperative behaviors and minority opinions. Decision Support Systems, 79:150-160, 2015 .

[45] Y.J. Xu, R. Patnayakuni, and H.M. Wang. The ordinal consistency of a fuzzy preference relation, Information Sciences, 224:152-164, 2013.

[46] Z.S. Xu and J. Chen. Some models for deriving the priority weights from interval fuzzy preference relations. European Journal of Operational Research, 184(1):266-280, 2008.

[47] R.R. Yager. On ordered weighted averaging aggregation operators in multicriteria decision making. IEEE Transactions on Systems, Man, and Cybernetics, 18:183-190, 1988.

[48] R.R. Yager. On the retranslation process in Zadeh's paradigm of computing with words. IEEE Transactions on Systems, Man and Cybernetics, Part B: Cybernetics, 34(2):1184-1195, 2004.

[49] L.A. Zadeh. Fuzzy logic = computing with words. IEEE Transactions on Fuzzy Systems, 4(2):103-111, 1996.

[50] L.A. Zadeh. From Computing with Numbers to Computing with WordsFrom Manipulation of Measurements to Manipulation of Perceptions, pages 35-68. In: Computing with Words, P.P. Wang (Ed.). Wiley Series on Intelligent Systems. John Wiley and Sons, 2001. 


\section{Appendix}

\section{The proof of Proposition 1}

In model $P$, constraints (23)and (24)guarantee that $F^{2 k} \in N_{\widetilde{V k}}$, constraint (25) guarantees that $C L\left(F^{1 k}\right)=1$, constraints (26) and (27) guarantee that $F^{1 k} \in N_{\widetilde{V^{k}}}$, constraint (28) guarantees that $a_{i} \in I N S^{k}\left(s_{i}\right)$, and constraints(29) and (30) guarantee that $I N S^{k}$ is ordered.

This completes the proof of Proposition 1.

\section{The proof of Proposition 2}

The properties of the PTLOWA operator (1)-(4) are proved as follows,

(1) Let $q_{1}=\min _{\alpha \in\{1, \ldots, m\}} \sum_{k=1}^{m} w_{k} I N S^{k}\left(r_{\alpha}\right), q_{2}=\max _{\alpha \in\{1, \ldots, m\}} \sum_{k=1}^{m} w_{k} I N S^{k}\left(r_{\alpha}\right)$, and $q=\sum_{k=1}^{m} w_{k} I N S^{k}\left(r_{k}\right)$.

Since $I N S$ is ordered, it is clear that $q_{1} \leq q \leq q_{2}$. So, we can get $I N S^{k-}\left(q_{1}\right) \leq I N S^{k-}(q) \leq I N S^{k-}\left(q_{2}\right)$ for $k=1,2, \ldots, m$, and $\left(I N S^{1-}\left(q_{1}\right), I N S^{2-}\left(q_{1}\right), \ldots, I N S^{m-}\left(q_{1}\right)\right)^{T} \leq P T L O W A_{W}\left(r_{1}, r_{2}, \ldots, r_{m}\right) \leq$ $\left(I N S^{1-}\left(q_{2}\right), I N S^{2-}\left(q_{2}\right), \ldots, I N S^{m-}\left(q_{2}\right)\right)^{T}$, which completes the proof of property (1).

(2) Since $r_{k} \sim r_{t}$ for $k=1,2, \ldots, m$, it follows that $I N S^{k}\left(r_{k}\right)=I N S^{t}\left(r_{t}\right)$, and $z_{t}=w_{1} \times I N S^{1}\left(r_{1}\right)+w_{2} \times I N S^{2}\left(r_{2}\right)+\ldots+w_{m} \times I N S^{m}\left(r_{m}\right)=w_{1} \times$ $I N S^{\sigma(1)}\left(r_{\sigma(1)}\right)+w_{2} \times I N S^{\sigma(2)}\left(r_{\sigma(2)}\right)+\ldots+w_{m} \times I N S^{m}\left(r_{\sigma(m)}\right)=I N S^{t}\left(r_{t}\right)$. So, PTLOW $A_{W}\left(r_{1}, r_{2}, \ldots, r_{m}\right)=\left(I N S^{1-}\left(z_{t}\right), I N S^{2-}\left(z_{t}\right), \ldots, I N S^{m-}\left(z_{t}\right)\right)^{T}=$ $\left(r_{1}, r_{2}, \ldots, r_{m}\right)^{T}$, which completes the proof of property $(2)$.

(3) Let PTLOW $A_{W}\left(r_{1}, r_{2}, \ldots, r_{m}\right)=\left(I N S^{1-}(z), I N S^{2-}(z), \ldots, I N S^{m-}(z)\right)^{T}$, where $z=w_{1} \times I N S^{\sigma(1)}\left(r_{\sigma(1)}\right)+w_{2} \times I N S^{\sigma(2)}\left(r_{\sigma(2)}\right)+\ldots+w_{m} \times I N S^{\sigma(m)}\left(r_{\sigma(m)}\right)$. Let PTLOW $A_{W}\left(r_{1}^{\prime}, r_{2}^{\prime}, \ldots, r_{m}^{\prime}\right)=\left(I N S^{1-}\left(z^{\prime}\right), I N S^{2-}\left(z^{\prime}\right), \ldots, I N S^{m-}\left(z^{\prime}\right)\right)^{T}$, where $z^{\prime}=w_{1} \times I N S^{\sigma(1)}\left(r_{\sigma(1)}^{\prime}\right)+w_{2} \times I N S^{\sigma(2)}\left(r_{\sigma(2)}^{\prime}\right)+\ldots+w_{m} \times I N S^{\sigma(m)}\left(r_{\sigma(m)}^{\prime}\right)$. Because $\left(r_{1}^{\prime}, r_{2}^{\prime}, \ldots, r_{m}^{\prime}\right)$ is any permutation of $\left(r_{1}, r_{2}, \ldots, r_{m}\right)$, so we have PTLOW $A_{W}\left(r_{1}^{\prime}, r_{2}^{\prime}, \ldots, r_{m}^{\prime}\right)=P T L O W A_{W}\left(r_{1}, r_{2}, \ldots, r_{m}\right)$, which completes the proof of property (3).

(4) Let PTLOW $A_{W}\left(r_{1}, r_{2}, \ldots, r_{m}\right)=\left(I N S^{1-}(z), I N S^{2-}(z), \ldots, I N S^{m-}(z)\right)^{T}$, where $z=w_{1} \times I N S^{\sigma(1)}\left(r_{\sigma(1)}\right)+w_{2} \times I N S^{\sigma(2)}\left(r_{\sigma(2)}\right)+\ldots+w_{m} \times I N S^{m}\left(r_{\sigma(m)}\right)$.

Let PTLOW $A_{W}\left(r_{1}^{\prime}, r_{2}^{\prime}, \ldots, r_{m}^{\prime}\right)=\left(I N S^{1-}\left(z^{\prime}\right), I N S^{2-}\left(z^{\prime}\right), \ldots, I N S^{m-}\left(z^{\prime}\right)\right)^{T}$, where $z^{\prime}=w_{1} \times I N S^{1}\left(r_{\sigma(1)}^{\prime}\right)+w_{2} \times I N S^{2}\left(r_{\sigma(2)}^{\prime}\right)+\ldots+w_{m} \times I N S^{m}\left(r_{\sigma(m)}^{\prime}\right)$.

Since $r_{k} \succ r_{k}^{\prime}$ and $I N S$ is ordered, it follows that $r_{\sigma(k)} \succ r_{\sigma(k)}^{\prime}$ and $z>z^{\prime}$, 
then we can get

$\operatorname{PTLOW} A_{W}\left(r_{1}, r_{2}, \ldots, r_{m}\right)=\left(I N S^{1-}(z), I N S^{2-}(z), \ldots, I N S^{m-}(z)\right)^{T}>$ $\operatorname{PTLOW} A_{W}\left(r_{1}^{\prime}, r_{2}^{\prime}, \ldots, r_{m}^{\prime}\right)=\left(I N S^{1-}\left(z^{\prime}\right), I N S^{2-}\left(z^{\prime}\right), \ldots, I N S^{m-}\left(z^{\prime}\right)\right)^{T}$.

This completes the proof of Proposition 2. 